\title{
A systematic review of feeding practices among postoperative patients: is practice in-line with evidence-based guidelines?
}

Running title: Postoperative feeding in habitual practice

\section{Author information:}

1. Megan Rattray, School of Allied Health Sciences; and Menzies Health Institute Queensland, Griffith University, Gold Coast Campus, QLD 4222, Australia.

2. Shelley Roberts, NHMRC Centre of Research Excellence in Nursing; and Menzies Health Institute Queensland, Griffith University, Gold Coast Campus, 4222, Australia.

3. Andrea Marshall, School of Nursing and Midwifery; NHMRC Centre of Research Excellence in Nursing Interventions for Hospitalized Patients (NCREN); and Menzies Health Institute Queensland, Griffith University, Gold Coast Campus, 4222, Australia.

4. Ben Desbrow, School of Allied Health Sciences; and Menzies Health Institute Queensland, Griffith University, Gold Coast Campus, QLD 4222, Australia.

Correspondence: Megan Rattray, School of Allied Health Sciences, Griffith University, Gold Coast Campus, QLD 4222, Australia. E-mail: megan.rattray@griffithuni.edu.au

Key words: Early oral feeding; perioperative care; postoperative care; early recovery after surgery

Authorship: All authors contributed to study design and conception; data collection; data analysis and interpretation; and drafting, reviewing and approving the final version of the manuscript.

Conflict of interest and funding disclosure: The authors declare no conflicts of interests. This study received internal institutional support only. 


\title{
A systematic review of feeding practices among postoperative patients: is practice in-line with evidence-based guidelines?
}

\begin{abstract}
Background: Early oral feeding after surgery is best practice among adult, non-critically ill patients. Evidenced-based guidelines (EBG) recommend commencing liquid and solid feeding within 24 hours of surgery to improve patient (e.g. reduced morbidity) and hospital (e.g. reduced length of stay) outcomes. Whether these EBG are adhered to in usual clinical practice remains unknown. The aim of this study was to identify the time to commencement of first oral feed (liquid or solid) and first solid feed among postoperative, non-critically ill, adult patients.
\end{abstract}

Methods: MEDLINE, CINAHL, SCOPUS and Web of Science databases were searched from inception to June 2016 for observational studies reporting liquid and/or solid feeding practices among postoperative patients. Studies reporting a mean/median time to first feed or first solid feed within 24 hours of surgery or where $\geq 75 \%$ of patients were feeding by postoperative day one were deemed in-line with EBG.

Results: Of 5826 articles retrieved, 29 studies were included. Only 40\% and 22\% of studies reported time to first feed and time to first solid feed in-line with EBG, respectively. Clear and free liquids were the first diet types commenced in $86 \%$ of studies. When solids were commenced, $44 \%$ of studies reported using various therapeutic diet types (e.g. light) prior to the commencement of a regular diet. Patients that underwent gastrointestinal procedures appeared more likely to experience delayed postoperative feeding.

Conclusions: Our findings demonstrate a gap between postoperative feeding evidence and its practical application. This information provides a strong rationale for interventions targeting improved nutritional care following surgery. 


\section{Introduction}

The traditional postoperative feeding approach dictates fasting patients until the return of bowel function (e.g. passage of flatus and/or stool or bowel sounds) ${ }^{(1)}$. This approach was designed to avoid paralytic ileus (leading to vomiting, aspiration pneumonia, and wound dehiscence) and/or anastomotic leakage thought to occur if fed prematurely ${ }^{(1-3)}$. However, there is little evidence to suggest that these adverse outcomes are likely to occur, particularly following non-gastrointestinal surgeries ${ }^{(4-6)}$. Traditional postoperative feeding results in substantial periods of avoidable inadequate nutritional intake among patients who may already be at nutritional risk, and who require optimal nutrition for recovery and prevention of complications ${ }^{(4)}$. Malnutrition is a prevalent problem among surgical patients due to factors preceding (e.g. diseased state, preoperative dietary practices) and following surgery (e.g. postoperative symptoms and dietary practices) ${ }^{(7,8)}$.

In contrast to traditional beliefs, recent evidence indicates early oral or enteral feeding (i.e. within 24 hours after surgery) is safe and beneficial to adult, non-critically ill, postoperative patients $(1,5,6,9-18)$. Early feeding has been associated with faster recovery of intestinal function (i.e. resolution of ileus) ${ }^{(5,6,9,10,19,20)}$, reduced morbidity (e.g. less infectious complications and improved wound healing and immunity) $(5,11-13,15,21,22)$ and improved quality of life (e.g. patient satisfaction and ambulation) (12, 16-19) among various surgical populations such as upper and lower gastrointestinal, obstetric and gynaecological patients. In addition, a rapid transition back to solid food is important for reducing the risk of malnutrition and its associated consequences such as infections ${ }^{(23)}$, falls ${ }^{(24,25)}$, pressure injuries ${ }^{(26)}$, morbidity and mortality ${ }^{(27)}$. Improved patient-related outcomes translate into shorter lengths of hospitalisation and reduced health care costs $(5,9,11,13-16,28)$. As such, there are many potential benefits to the rapid reintroduction of nutrition following surgery.

Over the past decade, early oral feeding practice recommendations have been incorporated into evidenced-based post-operative care guidelines for gynaecologic (2), hepatic (29), pancreaticoduodenal ${ }^{(30)}$, gastric ${ }^{(31)}$, and colorectal, rectal and pelvic patients ${ }^{(32-34)}$. In general, these guidelines recommend liquid feeding to recommence within 24 hours, or ideally within 4 hours following surgery in low risk patient populations (e.g. lower gastrointestinal). Solid feeding is then suggested to commence within 24 hours of surgery ${ }^{(2,}$ 29-34). 
Despite clear guidelines, it is well known that the adoption of research findings into clinical practice is often a slow and onerous process, with evidenced-based research taking up to two decades to establish as habitual practice ${ }^{(35)}$. Further, studies show $30-40 \%$ of patients do not receive healthcare in accordance with evidence-based knowledge ${ }^{(36)}$. Considering traditional practices are difficult to change ${ }^{(37,38)}$, investigations into whether this is the case for early commencement of feeding among non-critically ill, adult, postoperative patients are warranted.

The aim of the present study is to examine feeding practices in postoperative patients by performing a systematic review of the available evidence. Considering transient improvements are often seen with intervention programs in clinical practice ${ }^{(39)}$, this evidence will be reviewed in the context of habitual practice only (i.e. absence of recent intervention programs). Findings from this study will clarify whether delayed postoperative fluid and/or solid feeding practices persist and to what extent among various postoperative groups. This information will inform whether (and where) interventions are required to bridge the gap between knowledge and practice to improve patient and health care-related outcomes. As such, the specific aims of this review are to identify in the context of habitual clinical practice:

1. The time to and type of first feed (liquid or solid) commenced among non-critically ill, adult, post-operative patients;

2. The time to and type of first solid feed commenced among non-critically ill, adult, post-operative patients; and

3. Whether specific postoperative patient groups are at greater risk of delayed feeding than others. 


\section{Methods}

The methodology of this review was devised in accordance with the Meta-Analyses and Systematic Reviews of Observational Studies Guidelines (MOOSE) ${ }^{(40)}$ and registered at the International Prospective Register for Systematic Reviews (identification code: CRD 42016052832).

\subsection{Search methods}

Potentially eligible studies were identified by searching the online databases MEDLINE (1965-2016), Cumulative Index of Nursing and Allied Health Literature (CINAHL, 19852016), SCOPUS (1977-2016) and Web of Science (1956-2016), using a Boolean search strategy developed in collaboration with a librarian. The search strategy involved using the AND operator to link keywords used for the population (e.g. postoperativ* OR surg* OR hospital), exposure (“nil by mouth” OR “clear fluids” OR “oral feeding”) and outcome (time OR practice OR frequency) of interest. Truncation was used where applicable to capture variation in word terminology (e.g. postoperativ*: postoperative, postoperatively) and enclosed quotation marks were used to search for exact phrases. The search was not limited to language, nor restricted by any other means, with the exception of limits set on research fields in Web of Science (restricted to surgery, cardiovascular system cardiology, gastroenterology hepatology, respiratory system, general internal medicine, oncology, otorhinolaryngology, urology nephrology, anaesthesiology, geriatrics gerontology, emergency medicine, nutrition dietetics, nursing, obstetrics gynaecology, rehabilitation and physiology). To maximise retrieval of eligible evidence, forward and backward citation tracking was performed on all included studies.

\subsection{Selection criteria}

Original studies were deemed eligible if they conformed to the pre-determined inclusion criteria (Table 1). Observational designs in which one or more of the following measures were reported (or could be calculated) in non-critically ill, adult, postoperative patients, were considered for inclusion: (1) time to first feed (liquid or solid) (2) fasting/nil-by-mouth duration, (3) time to first solid feed, or (4) proportion of patients feeding (liquid or solid) postoperatively. Articles were deemed eligible regardless if these measures were reported as primary or secondary outcomes. Survey designs were excluded given surveyed responses among medical professionals result in high bias ${ }^{(41)}$. Only before data were extracted from before-and-after studies (e.g. case-control or cohort studies), considering our aim was to 
assess habitual clinical practice and intervention strategies often result in transient improvements ${ }^{(39)}$. No restrictions were set on postoperative patient type (e.g. orthopaedic, upper gastrointestinal or lower gastrointestinal), considering this is the first review to investigate feeding practices among postoperative patients and consequently identify high risk groups. Finally, only studies available in full text and English were included.

\subsection{Eligibility screening}

All results retrieved from electronic databases and hand searching (i.e. forward and backward citation tracking) were downloaded and stored in EndNote version X7.5 (Thomson Reuters, New York, United States). Duplicate results were identified in EndNote and removed. Title and/or abstracts of all results were independently screened by one reviewer according to the section criteria outlined in Table 1. Full text copies of potentially eligible studies were scrutinised by two authors independently, ensuring consistency and reliability of results. In the event that data was not adequately reported or outcome measures of interest were mixed with exclusion criteria (e.g. feeding data for surgical and non-surgical ${ }^{(42)}$ or oral/enteral and parenteral populations presented together ${ }^{(7,43)}$, the corresponding author was contacted via email in an attempt to retrieve required data. Any uncertainties regarding potential study eligibility were resolved via discussion with all contributing authors.

\subsection{Data extraction}

Data extraction involved collecting study characteristics (author, setting, design, time/s data were collected), patient characteristics (American Society of Anaesthesiologists (ASA) score, age, gender), procedural details (location, approach, aesthetic technique, operation time), outcomes measures, method and type of first feed administered and postoperative feeding protocol details from eligible studies. First feed and first solid feed data were extracted in the form of mean (and standard deviation) or median (and IQR or range) time to feeding (days or hours) and/or percentage of patients feeding per postoperative day. Where studies reported data in both forms (i.e. median/mean and proportion), all relevant data were extracted. Where specified, details regarding postoperative care guidelines were extracted and recorded as evidence-based (i.e. part of ERAS, FT programs) or traditionally-based, as outlined in studies. 
Table 1. Selection criteria and definitions

\begin{tabular}{|c|c|c|}
\hline $\begin{array}{l}\text { Study } \\
\text { characteristics }\end{array}$ & Inclusion criteria & Exclusion criteria \\
\hline Design & -Observational studies (e.g. cohort, case-control, audits, cross-sectional) & $\begin{array}{l}\text {-Surveys } \\
\text {-Studies which impose a nutrition prescription, protocol or fasting duration } \\
\text { which is standardised as part of a clinical trial } \\
\text {-Research publications that are reviews of the literature or clinical practice } \\
\text { guidelines }\end{array}$ \\
\hline Sample/ setting & $\begin{array}{l}\text {-Adult patients } \\
\text {-Postoperative patients (any type) receiving feeding via the GIT } \\
\text {-Non-critically ill patients (i.e. not burns, cervical spine injuries, blunt } \\
\text { trauma or mechanically ventilated patients and where }<10 \% \text { of study } \\
\text { population had }<1 \text { day stay in an ICU) } \\
\text {-Any hospital setting (e.g. single-/multi-centre) }\end{array}$ & $\begin{array}{l}\text {-Exclusive inclusion of (or relevant data mixed and cannot be separated } \\
\text { from): } \\
\text {-preoperative or non-surgical patients } \\
\text {-patients }<18 \text { years of age or prescribed parenteral nutrition } \\
\text {-Animals }\end{array}$ \\
\hline Intervention & $\begin{array}{l}\text {-Baseline data (i.e. pre-intervention) from ERAS, FT or early feeding } \\
\text { interventions }\end{array}$ & $\begin{array}{l}\text {-Recent implementation of an ERAS, FT or early feeding program (i.e. data } \\
\text { collected within } 2 \text { years of implementation) or baseline data not reported } \\
\text {-Primarily aimed to assess the efficacy, safety or outcomes of a 'new' (e.g. } \\
\text { laparoscopic) and/or 'old' (e.g. open) surgical approach }\end{array}$ \\
\hline $\begin{array}{l}\text { Outcome } \\
\text { measures }\end{array}$ & $\begin{array}{l}\text {-Fasting/nil-by-mouth (i.e. the interval }{ }^{\mathrm{a}} \text { between } \mathrm{D} 0^{\mathrm{b}} \text { and time to first oral } \\
\text { or enteral feed) } \\
\text {-Time to first feed (i.e. the interval }{ }^{\mathrm{a}} \text { between } \mathrm{D} 0^{\mathrm{b}} \text { and time to first oral or } \\
\text { enteral feed) } \\
\text {-Time to first solid intake (i.e. the interval }{ }^{\mathrm{a}} \text { between } \mathrm{D} 0^{\mathrm{b}} \text { and time to first } \\
\text { solid intake) } \\
\text {-Proportion }{ }^{\mathrm{d}} \text { of patients feeding (liquid or solid) postoperatively }\end{array}$ & $\begin{array}{l}\text {-First feed and/or solid feed data not reported or cannot be calculated from } \\
\text { graphs } \\
\text {-Exclusive investigation of tolerance to first feed/ first solid feed }\end{array}$ \\
\hline Other & $\begin{array}{l}\text {-Available in full and English text } \\
\text {-Any publication timeframe }\end{array}$ & -Duplicate findings \\
\hline
\end{tabular}

[ERAS: early recovery after surgery; FT: fast track; GIT: gastrointestinal tract; ICU: intensive care unit]

${ }^{\text {aHours or days }}$

bDay of surgery

'Only feeds containing macronutrients were included (i.e. where water were specified as first feed, this data was excluded)

${ }^{\mathrm{d}}$ Number of patients divided by cohort number 


\subsection{Quality appraisal}

The methodological quality of eligible studies was assessed by two reviewers, using an adapted quality assessment tool, designed to critically appraise cross-sectional, case-control, and cohort designs (Appendix 1). This tool was developed following semi-thematic analysis of questions asked across the Critical Appraisal Skills Programme (CASP) and Joanna Briggs checklists for case-control, cohort and analytical cross-sectional studies ${ }^{(44,45)}$. Quality scores were based on one of four categorical possibilities (no, yes, unsure, not applicable) consistent with the CASP and Joanna Briggs checklists for observational studies ${ }^{(44,45)}$. Only the methods and results related to pre-data for case-control and cohort studies were assessed for quality, considering this data was solely extracted. The overall quality of each study was calculated by dividing the number of yes answers by applicable questions. Any disagreements or uncertainties regarding quality assessment were resolved via discussion with all contributing author.

\subsection{Data synthesis/analysis}

Data were entered into Excel 2010 (Microsoft Office, New Mexico, United States) and tabulated according to postoperative patient population: (1) only lower gastrointestinal and small bowel (rectum, anus, colon and small bowel procedures); (2) only upper gastrointestinal (liver, pancreas, stomach or oesophageal procedures); (3) orthopaedic (hip or knee procedures); (4) obstetric and gynaecological (uterus, vagina, ovaries, and fallopian tubes procedures); and (5) abdominal (various or unspecified abdominal procedure types, e.g. the gall balder, small bowel, colon, and stomach). Study populations were considered 'normal' to 'mildly' healthy where $\geq 75 \%$ of the cohort had an American Society of Anesthesiologists (ASA) score of 1 or $2^{(46)}$. In instances where studies presented multiple data sets $(n=3)(7,47,48)$, each data set was treated as individual investigations. Proportional data were reported as either (1) number of patients commencing feeding per postoperative day or (2) total (i.e. accumulative) number of patients feeding per postoperative day. For consistency and comparative purposes, all proportional data is presented as the latter. Similarly, nil-by-mouth and mean/median time to first feed were collapsed into one measure (mean/median time to first feed), considering they signify the same outcome. Raw time to feed data retrieved from studies were tested for normality and subsequently presented as either mean(SD) or median(IQR) ${ }^{(43,49)}$. For one study, a pseudo-value of 5 days was used where eligible patients ( $n=58,2$ patients were excluded from analysis; 1 was $<18$ years of age 
and 1 received TPN as first feed) had not commenced feeding by postoperative day 5 (data collection ceased after this day) $(n=4)$ to calculate median time to first feed ${ }^{(49)}$. One study grouped feeding data (proportion) based on their definition of patients who underwent 'non fast track' and 'fast track' principles; however, this data was re-combined for analysis (50). Studies reporting a mean/median time to first feed or first solid feed within 24 hours of surgery or where $\geq 75 \%$ patients were feeding by postoperative day one (D1) were deemed inline with evidence-based guideline recommendations. This definition was chosen as commencing feeding within 24 hour of surgery (i.e. by D1) is the upper timeframe recommended across all postoperative care guidelines ${ }^{(2,29-34)}$. 


\section{Results}

Figure 1 provides details on the search strategy undertaken, including potential study identification, screening, eligibility and inclusion. Overall, 29 papers met the eligibility criteria and were included in the review $(7,43,47-73)$.

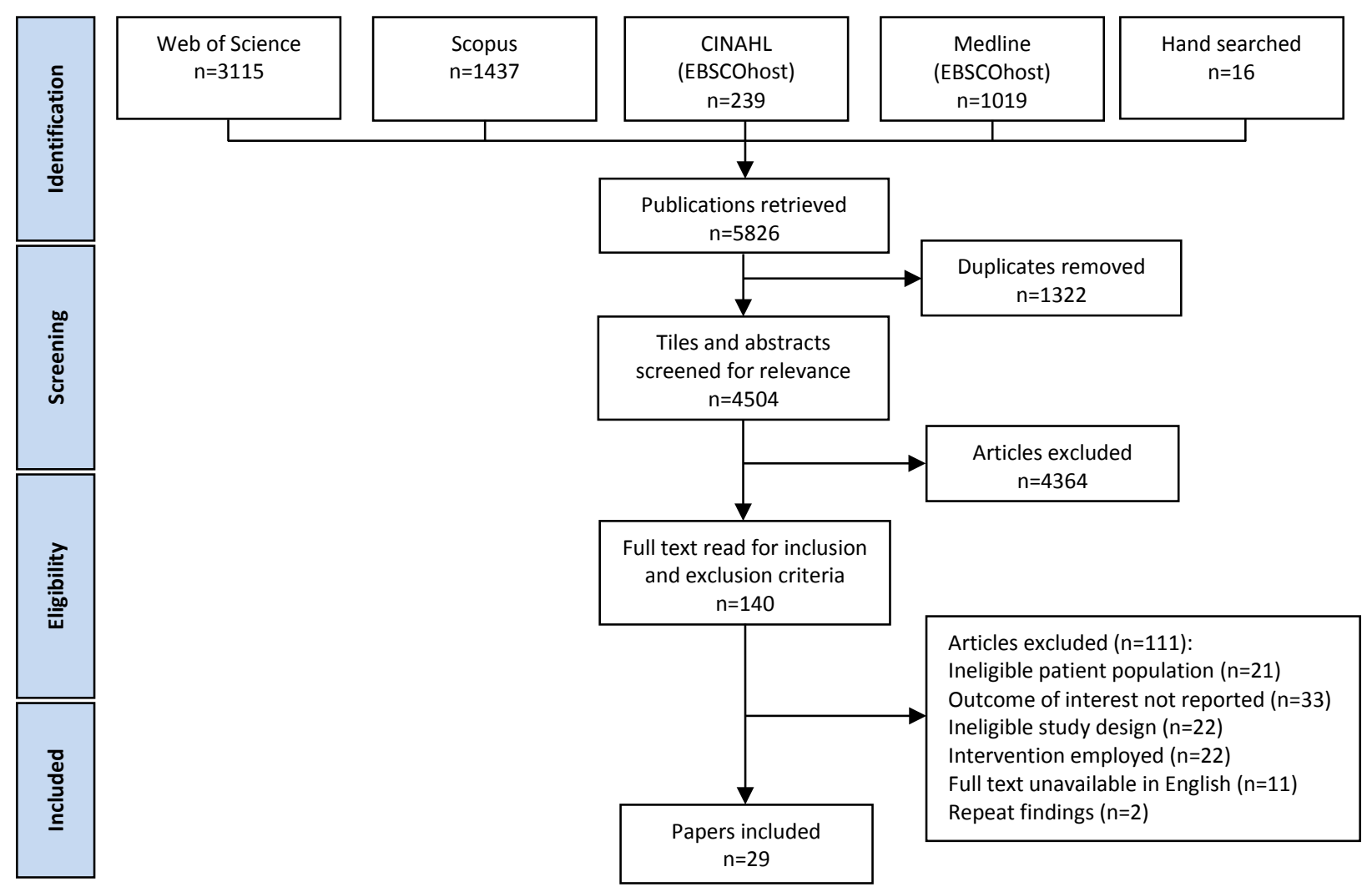

Figure 1. Selection of articles for review

\subsection{Description of study and patient characteristics}

Study and patient characteristics of included investigations are described in Table 2. The majority of included investigations were case-control or cohort studies using a pre- and postintervention design $(n=20,69 \%)$. The majority of studies were undertaken at a single site $(n=23,79 \%)$ and collected data within the last decade $(n=22,76 \%)$.

Overall, 19,046 patients were included in this review. The majority of studies $(n=19,66 \%)$ had mean/median populations aged between 60 to 70 years. Of the studies which reported ASA scores ( $n=16,55 \%)$, the majority ( $n=10,63 \%)$ investigated 'normal' to 'mildly' healthy populations. A range of patient populations were investigated, including patients undergoing lower GI ( $n=12)$, upper GI ( $n=5)$, orthopaedic $(n=3)$, obstetric and gynaecological $(n=5)$ or abdominal $(n=5)$ procedures. Of the studies specifying details on procedural approach (i.e. 
Table 2. Study and patient characteristics and outcomes of interest

\begin{tabular}{|c|c|c|c|c|c|c|}
\hline \multirow[t]{2}{*}{ Reference } & \multirow[t]{2}{*}{ Methods } & \multirow[t]{2}{*}{ Setting } & \multirow[t]{2}{*}{ Sample ${ }^{a}$} & \multirow[t]{2}{*}{ Procedure details } & \multicolumn{2}{|c|}{ Outcomes $^{a}$} \\
\hline & & & & & First feed & First solid feed \\
\hline $\begin{array}{l}\text { Alcántara-Moral et al., } \\
2014\end{array}$ & $\begin{array}{l}\text { Cross-sectional, data collected prospectively } \\
\text { (May 2008-Dec 2010) }\end{array}$ & $M C(n=25)$, Spain & 363 colorectal: $68.7 y ; 55 \%$ M ; 63\% ASA $1 \& 2$ & $\begin{array}{l}\text { Open (51\%) right hemicolectomy }(44 \%) \text {, } \\
\text { sigmoidectomy (37\%) or left hemicolectomy (11\%) }\end{array}$ & $\begin{array}{l}<12 \mathrm{~h}: 31 \%, \mathrm{~F} \\
\text { D1:58\%, }, \mathrm{F} \\
\text { D2:70\%, F } \\
\text { D3:87\%, } \mathrm{F} \\
\text { D4:94\% }{ }^{\mathrm{b}}, \mathrm{F}\end{array}$ & $\begin{array}{l}\text { D0:0\% } \%^{\mathrm{b}}, \mathrm{S} \\
\text { D1:5\% } \%^{\mathrm{b}}, \mathrm{S} \\
\text { D2:40\% } \\
\text { D3: } 68 \%^{\mathrm{b}}, \mathrm{S} \\
\text { D4:81\% } \\
\text { D }, \mathrm{S}\end{array}$ \\
\hline Benhamou et al., 2002 & $\begin{array}{l}\text { Before-and-after, before data collected } \\
\text { prospectively (Aug-Oct 1996) }\end{array}$ & SC, France & 53 caesarean: patient characteristics $\mathrm{n} / \mathrm{s}$ & Elective $\left(29 \% m^{m}\right)$ caesarean & $\begin{array}{l}\text { D0:92\%, L } \\
\text { D1:100\%, L (89\%) \& R } \\
(11 \%)\end{array}$ & $\begin{array}{l}\text { D0:92\%, L } \\
\text { D1:100\%, L (89\%) \& R (11\%) }\end{array}$ \\
\hline Cestonaro et al., 2014 & $\begin{array}{l}\text { Cross-sectional, data collected prospectively } \\
\text { (Feb-Jul 2012) }\end{array}$ & $\begin{array}{l}\text { SC, university hospital, } \\
\text { Brazil }\end{array}$ & 135 abdominal: $(76 \%)<60 y ; 39 \%$ M; $93 \%$ ASA $1 \& 2$ & $\begin{array}{l}\text { Elective laparoscopic (most } / y) \text { hernioplasty }(43 \%), \\
\text { cholecystectomy (35\%) \& Nissen fundoplication } \\
(10 \%)\end{array}$ & 15.7(1.7-90.4)h $\mathrm{h}^{\mathrm{d}}, \mathrm{OR}$ & $\mathrm{n} / \mathrm{s}$ \\
\hline Christelis et al., 2015 & $\begin{array}{l}\text { Before-and-after, before data collected } \\
\text { prospectively (Mar-Sep 2012) }\end{array}$ & $M C(n=3), A U$ & 412 orthopaedic: $68 \pm 11 y ; 40 \%$ M; $56 \%$ ASA $1 \& 2$ & Elective hip (52\%), knee (48\%) or revision (6\%) & $3.2(2-5) \mathrm{h}^{\mathrm{e}}, \mathrm{F}$ & 7.0(4.3-15)he, S \\
\hline Conchin et al., $2013^{c}$ & $\begin{array}{l}\text { Audit (cross-sectional), data collected } \\
\text { retrospectively (Nov 2010-May 2011) }\end{array}$ & $\begin{array}{l}\text { SC, tertiary teaching } \\
\text { hospital, AU }\end{array}$ & 22 gynaecological: $63.7 \pm 12.3 y r s ; 50 \%$ M & Open (73\%) gynaecological or urological & $\begin{array}{l}1(0-3) d^{e}, C F(73 \%) \& F F \\
(27 \%) \\
\text { D0:0\%, F } \\
\text { D1:41\%, F }\end{array}$ & $\begin{array}{l}4(3-6) d^{e}, \text { P }(96 \%) \\
\text { D0:0\%, P } \\
\text { D1:0\%, P } \\
\text { D2:13\%, P } \\
\text { D3:35\%, P } \\
\text { D4:52\%, P }\end{array}$ \\
\hline $\begin{array}{l}\text { de Aguilar-Nascimento et } \\
\text { al., } 2008\end{array}$ & $\begin{array}{l}\text { Before-and-after study, before data collected } \\
\text { prospectively (Jan-Jun 2005) }\end{array}$ & $\begin{array}{l}\text { SC, tertiary university } \\
\text { hospital, Brazil }\end{array}$ & 78 abdominal: $44(18-85) y^{d} ; 62 \% \mathrm{M}$ & $\begin{array}{l}\text { Elective cholecystectomy }(32 \%) \text {, herniography }(23 \%) \\
\text { or colorectal }(13 \%)\end{array}$ & $1(0-20) d^{d}, n / s$ & $\mathrm{n} / \mathrm{s}$ \\
\hline $\begin{array}{l}\text { de Aguilar-Nascimento et } \\
\text { al., } 2010\end{array}$ & $\begin{array}{l}\text { Historical cohort study, before data collected } \\
\text { retrospectively (Jan 2004-Jun 2005) }\end{array}$ & $\begin{array}{l}\text { SC, tertiary university } \\
\text { hospital, Brazil }\end{array}$ & 42 abdominal: $67.5(60-85) y^{d} ; 64 \%$ M; 93\% ASA $1 \& 2$ & $\begin{array}{l}\text { Elective colorectal (26\%), choloecistectomy (21\%) or } \\
\text { herniography }(19 \%)\end{array}$ & $1(0-10 d) d^{b}, n / s$ & $\mathrm{n} / \mathrm{s}$ \\
\hline de Groot et al., 2014 & $\begin{array}{l}\text { Quality improvement, before data collected } \\
\text { retrospectively (Jan 2007-Jan 2008) }\end{array}$ & $\begin{array}{l}\text { SC, tertiary hospital, the } \\
\text { Netherlands }\end{array}$ & 38 gynaecological : $62.7 \pm 12.6 y ;$ M 0\%; 92\% ASA $1 \& 2$ & $\begin{array}{l}\text { Abdominal surgery through a midline }(82 \%) \text { incision } \\
\text { for gynecologic malignancies }\end{array}$ & $\begin{array}{l}2(2-3) d^{e}, F \\
\text { D0: } 0 \%, F\end{array}$ & $\begin{array}{l}3(3-4) d^{e}, R \\
D 1: 0 \%\end{array}$ \\
\hline Deniau et al., 2016 & $\begin{array}{l}\text { Audit, before data collected prospectively } \\
\text { (Nov 2012-Feb 2013) }\end{array}$ & SC, France & 45 caesarean: $0 \% \mathrm{M}$ & Elective $(35.5 \%)$ caesarean section & $<6 \mathrm{~h} \mathrm{D0}: 31 \%, \mathrm{~F}$ & $<6 \mathrm{~h} \mathrm{D0}: 20 \%, \mathrm{~S}$ \\
\hline Egerod et al., 2010 & $\begin{array}{l}\text { Audit, data collected retrospectively (Aug } \\
\text { 2007-Jan 2008) }\end{array}$ & $\mathrm{MC}(\mathrm{n}=30)$, Denmark & 594 orthopaedic: $83(39-103) y^{d} ; 28 \%$ M & Hip fracture & $\mathrm{n} / \mathrm{s}$ & D1:66\%, R \\
\hline Francisco et al., 2015 & $\begin{array}{l}\text { Cross-sectional, data collected prospectively } \\
\text { (Jul-Nov 2014) }\end{array}$ & SC, Brazil & 65 surgical: $27-53 y$ (47.7\%); $26 \%$ M; $93.8 \%$ & $\begin{array}{l}\text { Elective cholecystectomy (47.7\%), papillotomy } \\
(10.8 \%) \text { or liver abscess drainage (10.8\%) }\end{array}$ & $\begin{array}{l}17.4(2.5-29.6) h^{d}, F \\
\text { D0:25\%, F; } \\
\text { D1:77\%, F } \\
\text { D2:89\%, F } \\
\text { D3:100\%, F }\end{array}$ & $\begin{array}{l}18.3(3.3-98.8) h^{d}, S \\
\text { D0:23\%, S } \\
\text { D1:77\%, S } \\
\text { D2:89\%, S } \\
\text { D3:100\%, S }\end{array}$ \\
\hline Garth et al., $2010^{c}$ & Cross-sectional, data collected retrospectively & $\begin{array}{l}\text { SC, tertiary teaching } \\
\text { hospital, } \mathrm{AU}\end{array}$ & $53^{8}$ colorectal: $65.2 \pm 14.3 y ; 69 \%$ M [DS1] & Elective open colorectal & $\begin{array}{l}1.2 \pm 1.2 \mathrm{~d}, \mathrm{n} / \mathrm{s} \text { [includes } \\
\mathrm{EN}]\end{array}$ & $\mathrm{n} / \mathrm{s}$ \\
\hline & (Mar 2006-Oct 2007) & $\begin{array}{l}\text { SC, tertiary teaching } \\
\text { hospital, AU }\end{array}$ & $35^{8}$ upper GI: $68.0 \pm 10.6 y ; 59 \%$ M [DS2] & Elective upper gastrointestinal & $\begin{array}{l}3.6 \pm 2.2 \mathrm{~d}, \mathrm{n} / \mathrm{s} \text { [includes } \\
\mathrm{EN}]\end{array}$ & $\mathrm{n} / \mathrm{s}$ \\
\hline Hardt et al., $2013^{c}$ & $\begin{array}{l}\text { Before-and-after, before data collected } \\
\text { retrospectively (Jan 2006-Dec 2007) }\end{array}$ & SC, Germany & 67 colorectal: $62 y^{\mathrm{e}} ; 69.4 \%$ M; $78 \%$ ASA $1 \& 2$ & $\begin{array}{l}\text { Elective open }(67 \%) \text { rectal resection with protective } \\
\text { ileostomy placement }\end{array}$ & $1.4 \pm 1.2 \mathrm{~d}, \mathrm{FF}$ & $\begin{array}{l}3.2 \pm 2.7 \mathrm{~d}, \mathrm{~S} \\
\mathrm{D} 1: 29 \%, \mathrm{~S} \\
\mathrm{D} 2: 52 \%, \mathrm{~S} \\
\end{array}$ \\
\hline Hilal et al., 2013 & $\begin{array}{l}\text { Case-control, before data collected } \\
\text { retrospectively (May 2009-Aug 2010) }\end{array}$ & $\begin{array}{l}\text { SC, university tertiary } \\
\text { hospital, UK }\end{array}$ & $\begin{array}{l}24 \text { pancreaticoduodenal : } 70 \text { (61-76)y ; } 42 \% \text { M; } 88 \% \\
\text { ASA } 1 \& 2\end{array}$ & $\begin{array}{l}\text { Standardised upper abdominal J laparotomy, distal } \\
\text { gastrectomy and lymphadenectomy }\end{array}$ & $\mathrm{n} / \mathrm{s}$ & $9(6-12) d^{e}, S$ \\
\hline Hubner et al., 2015 & $\begin{array}{l}\text { Cohort, before data collected prospectively } \\
\text { (2010) }\end{array}$ & $\begin{array}{l}\text { SC, tertiary academic } \\
\text { centre, Switzerland }\end{array}$ & 50 colorectal: $67(55.5-75.3) \mathrm{y}^{\mathrm{e}} ; 50 \% \mathrm{M} ; 66 \%$ ASA $1 \& 2$ & Open (44\%) left colon (42\%) or rectum (26\%) & $\mathrm{D} 0: 10 \%^{\mathrm{b}}, \mathrm{FF}$ & $\mathrm{n} / \mathrm{s}$ \\
\hline Hui et al., 2013 & $\begin{array}{l}\text { Before-and-after, before data collected } \\
\text { prospectively (Jan-Dec 2005) }\end{array}$ & $\begin{array}{l}\text { SC, university teaching } \\
\text { hospital, USA }\end{array}$ & 87 colorectal: $57.5 y ; 37 \%$ M; 84\% ASA $1 \& 2$ & Elective (100\%) open (62\%) bowel resection & $\mathrm{D} 0: 34 \%, \mathrm{n} / \mathrm{s}$ & $\mathrm{n} / \mathrm{s}$ \\
\hline Imbelloni et al., 2014c & $\begin{array}{l}\text { Longitudinal, before data collected } \\
\text { prospectively (May-Aug 2001) }\end{array}$ & SC, Brazil & 83 orthopaedic: $78.96 \pm 10.43 y ; 36 \% \mathrm{M}$ & Corrective femoral fracture & $17 \pm 2 h, R$ & $17 \pm 2 h, R$ \\
\hline Kalogera et al., 2013 & $\begin{array}{l}\text { Cohort, before data was collected } \\
\text { retrospectively (Mar-Dec 2010) }\end{array}$ & SC, USA & $\begin{array}{l}235 \text { gynaecological: } 65.1(61-71.3) y^{e} ; 0 \% \text { M; 59\% ASA } \\
1 \& 2\end{array}$ & $\begin{array}{l}\text { Complex cytoreductive }(33 \%) \text {, surgical staging }(34 \%) \\
\text { or pelvic organ prolapse }(32 \%)\end{array}$ & $\mathrm{n} / \mathrm{s}$ & $5(3-7) d^{e}, R$ \\
\hline Kolozsvari et al., 2013 & $\begin{array}{l}\text { Case-control audit, data collected } \\
\text { retrospectively (Jan 2003-Aug 2010) }\end{array}$ & $\begin{array}{l}\text { SC, university teaching } \\
\text { hospital, Canada }\end{array}$ & 161 colorectal: $62.9 \pm 16 y, 53 \% \mathrm{M}, 75 \%$ ASA $1 \& 2$ & $\begin{array}{l}\text { Elective }(100 \%) \text { laparoscopic }(88 \%) \text { right } \\
\text { hemicolectomies }(45 \%) \text { or anterior resections (37\%) }\end{array}$ & $\mathrm{n} / \mathrm{s}$ & $3(2-3.5) d^{e}, S$ \\
\hline
\end{tabular}




\begin{tabular}{|c|c|c|c|c|c|c|}
\hline \multirow{4}{*}{ Kuwabara et al., 2011} & \multirow{4}{*}{$\begin{array}{l}\text { Cross-sectional, data collected retrospectively } \\
\text { (Jul-Dec 2007) }\end{array}$} & \multirow{4}{*}{$\begin{array}{l}\text { MC ( } n=837) \text {, academic and } \\
\text { community hospitals, } \\
\text { Japan }\end{array}$} & 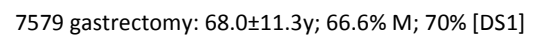 & Open partial gastrectomy & $5.3 \pm 3 d, O R$ & $\mathrm{n} / \mathrm{s}$ \\
\hline & & & 4111 gastrectomy: $67.7 \pm 10.5 y ; 73 \% \mathrm{M} ; 64 \%$ [DS2] & Open total gastrectomy & $6.7 \pm 5.4 \mathrm{~d}, \mathrm{OR}$ & $\mathrm{n} / \mathrm{s}$ \\
\hline & & & 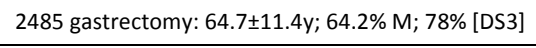 & Laparoscopic partial gastrectomy & $4.6 \pm 3.7 d, O R$ & $\mathrm{n} / \mathrm{s}$ \\
\hline & & & 290 gastrectomy: 64.8ะ11.1y; 67.2\% M; 81\% [DS4] & Laparoscopic total gastrectomy & $5.9 \pm 4.2 \mathrm{~d}, \mathrm{OR}$ & $\mathrm{n} / \mathrm{s}$ \\
\hline Labgaa et al., 2016 & $\begin{array}{l}\text { Before-and-after three phased study, before } \\
\text { data collected prospectively (<May 2011) }\end{array}$ & $\begin{array}{l}\text { SC, tertiary-referral } \\
\text { hospital, AU }\end{array}$ & 50 liver: 64(59-69)y*; 70\% M; 72\% ASA score $1 \& 2$ & Elective open (96\%) minor liver resection (54\%) & $\mathrm{DO}: 34 \%^{\mathrm{b}}, \mathrm{FF}$ & $\mathrm{D} 1: 5 \% \mathrm{~b}, \mathrm{R}$ \\
\hline Maessen et al., 2009 & $\begin{array}{l}\text { Before-and-after quality improvement } \\
\text { project. Before data collected retrospectively } \\
\text { (2004) }\end{array}$ & $M C(n=26)$, the Netherlands & 1126 colorectal: $65 y ; 51 \%$ M & Colorectal resection (details $\mathrm{n} / \mathrm{s}$ ) & $\mathrm{n} / \mathrm{s}$ & $\begin{array}{l}\text { Sd, R } \\
\text { D0:0\%+, R } \\
\text { D1:3\%+, R } \\
\text { D2:7\%, R } \\
\text { D3:23\%+, R } \\
\text { D4:40\%+, R } \\
\text { D5:56\%+, R }\end{array}$ \\
\hline Miller et al., 2014 & $\begin{array}{l}\text { Before-and-after study. Before data collected } \\
\text { retrospectively (Jan-Jun 2009) }\end{array}$ & $\begin{array}{l}\text { SC, tertiary teaching } \\
\text { hospital, USA }\end{array}$ & 99 colorectal: $56 \pm 15 y ; 43 \%$ M; 42\% ASA $1 \& 2$ & Laparoscopic $(40 \%)$, colon $(55 \%)$ or pelvic $(45 \%)$ & $\begin{array}{l}1.8 \pm 1.9 \mathrm{~d}, \mathrm{CF} \\
\mathrm{D0}: 15 \%, \mathrm{CF}\end{array}$ & $\mathrm{n} / \mathrm{s}$ \\
\hline \multirow{4}{*}{ Rettenmaier et al., 2014} & \multirow{4}{*}{$\begin{array}{l}\text { Cross-sectional, data collected retrospectively } \\
\text { (Jun 2009-Jun 2013) }\end{array}$} & \multirow{4}{*}{ SC, tertiary hospital, USA } & 52 colorectal: $62(29-91) y^{d}[D S 1]$ & Rectosigmoid resection & $\begin{array}{l}\text { D1-D2:38\%, OR } \\
\text { D3-D4:73, OR }\end{array}$ & $\mathrm{n} / \mathrm{s}$ \\
\hline & & & 43 colorectal: $62(29-91) y^{d}[D S 2]$ & Rectosigmoid resection and colectomy & $\begin{array}{l}\text { D1-D2:37\%, OR } \\
\text { D3-D4:70\%, OR }\end{array}$ & $\mathrm{n} / \mathrm{s}$ \\
\hline & & & 68 colorectal: 62(29-91)yd [DS3] & Proximal colectomy & $\begin{array}{l}\text { D1-D2:32\%, OR } \\
\text { D3-D4:59\%, OR }\end{array}$ & $\mathrm{n} / \mathrm{s}$ \\
\hline & & & 55 small bowel: 62(29-91)y $[$ [DS4] & Small bowel resection & $\begin{array}{l}\text { D1-D2:24\%, OR } \\
\text { D3-D4:65\%, OR }\end{array}$ & $\mathrm{n} / \mathrm{s}$ \\
\hline Rohatiner et al., $2012^{c}$ & $\begin{array}{l}\text { Cross-sectional, data collected prospectively } \\
\text { (Aug-Sep 2011) }\end{array}$ & $\begin{array}{l}\text { SC, urban teaching } \\
\text { hospital, USA }\end{array}$ & 58 small bowel \& colorectal: $57(1-96) y^{6} ; 53 \% \mathrm{M}$ & $\begin{array}{l}\text { Elective laparoscopic }(64 \%) \text { colorectal }(72 \%) \text { or small } \\
\text { bowel (28\%) }\end{array}$ & $\begin{array}{l}1(1-2) \text { de, CF }(81 \%), \text { EN } \\
\text { (7\%) } \\
\text { DO:9\%, CF (40\%), EN } \\
(40 \%) \\
\text { D1:62\%, CF }(94 \%) \\
\text { D2:78\%, CF }(100 \%) \\
\text { D3:86\%, CF }(80 \%) \\
\text { D4:91\%, CF }(100 \%)\end{array}$ & $\begin{array}{l}\text { D0:4\%, R (100\%) } \\
\text { D1:4\%, R (100\%) } \\
\text { D2:44\%, R (13\%) \& R/F (87\%) } \\
\text { D3:57\%, R (29\%), R/F (43\%) \& } \\
\text { other (28\%) } \\
\text { D4: 70\%; R (14\%), R/F (72\%) \& } \\
\text { other (14\%) }\end{array}$ \\
\hline Schwarzbach et al., 2011 & $\begin{array}{l}\text { Before-and-after, before data collected } \\
\text { retrospectively (Jan 2006-Dec 2007) }\end{array}$ & SC, Germany & 133 colorectal: $65 y^{\mathrm{e}} ; 52 \% \mathrm{M} ; 83 \%$ ASA $1 \& 2$ & $\begin{array}{l}\text { Elective laparoscopic }(51 \%) \text { sigmoid resection }(49 \%) \\
\text { or right hemicolectomy }(34 \%)\end{array}$ & $\mathrm{n} / \mathrm{s}$ & $\begin{array}{l}\mathrm{D} 1: 50 \%, \mathrm{~S} \\
\mathrm{D} 2: 59 \%, \mathrm{~S}\end{array}$ \\
\hline Selby et al., 2016 & $\begin{array}{l}\text { Before-and-after, before data collected } \\
\text { retrospectively (Jun 2007-Jun 2009) }\end{array}$ & SC, USA & 51 gastrectomy: 69(58-76)y $\mathrm{y}^{\mathrm{e}} 78 \% \mathrm{M}$ & Total open (82\%) gastrectomy & $4(3-5) d^{e}, C F$ & $6(5-7) d^{e}, P$ \\
\hline Thompson et al., 2012 & $\begin{array}{l}\text { Before-and-after, before data collected } \\
\text { prospectively (Jul-Dec 2009) }\end{array}$ & $M C(n=3), A U$ & 154 abdominal: $59 \pm 16 y ; 83 \% \mathrm{M}$ & $\begin{array}{l}\text { Elective open }(71 \%) \text { colorectal }(46 \%) \text {, gastric }(16 \%), \\
\text { small bowel }(13 \%) \text {, or hepatobiliary }(11 \%)\end{array}$ & $16(6-28) h^{e}, F$ & $2.8(1.1-5) \mathrm{d}^{\mathrm{e}}, \mathrm{S}$ \\
\hline $\begin{array}{l}\text { Zargar-Shoshtari et al., } \\
2008\end{array}$ & $\begin{array}{l}\text { Before-and-after, before data collected } \\
\text { retrospectively (Sep 2004-Sep 2005) }\end{array}$ & SC, New Zealand & 50 colorectal: $70.7(40-85) y^{\mathrm{d}} ; 56 \% \mathrm{M} ; 78 \%$ ASA $1 \& 2$ & $\begin{array}{l}\text { Elective open (86\%) right (58\%) or left (28\%) } \\
\text { hemicolocolectomy }\end{array}$ & $2(1-15) d^{d}, O R$ & $\mathrm{n} / \mathrm{s}$ \\
\hline
\end{tabular}

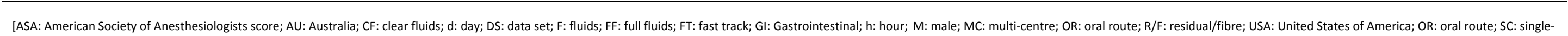
centre]

aUnless specified otherwise, data is presented as mean(SD)

Interpreted from graph

'Data received from authors

dMedian (range)

eMedian (IQR)

${ }^{f}$ Mean (range) 
open vs laparoscopic) ( $n=16,55 \%)$, the majority ( $n=9,56 \%)$ reported mainly (i.e. $>50 \%$ ) employing an open method; a proportion that was not influenced by only reviewing studies conducted within the last 5 years.

Table 3. First feed tabulated according to procedure

\begin{tabular}{|c|c|c|c|c|c|c|c|}
\hline \multirow[t]{2}{*}{ Study } & \multirow{2}{*}{$\begin{array}{l}\text { Mean } \pm S D \text { or } \\
\text { median (IQR) time } \\
\text { to first feed }\end{array}$} & \multicolumn{3}{|c|}{ Proportion } & \multicolumn{3}{|l|}{ Factors } \\
\hline & & Do & D1 & D2 & Origin & EBG & DC date/s \\
\hline \multicolumn{8}{|c|}{ Lower gastrointestinal (and small intestine) } \\
\hline Rohatiner et al., 2012 & $1(1-2) d^{b}$ & $9 \%$ & $62 \%$ & $78 \%$ & USA & $\mathrm{n} / \mathrm{s}$ & 2011 \\
\hline Garth et al., 2010 [DS1] & $1.2 \pm 1.2 \mathrm{~d}$ & & & & $A U$ & $\mathrm{n} / \mathrm{s}$ & $2006-2007$ \\
\hline Hardt et al., 2013 & $1.4 \pm 1.2 \mathrm{~d}$ & & & & Europe & Y & 2006-2007 \\
\hline Miller et al., 2014 & $1.8 \pm 1.9 \mathrm{~d}$ & $15 \%$ & & & USA & $\mathrm{N}$ & 2009 \\
\hline Zargasr-Shoshtari et al., 2008 & $2(1-15) d^{a}$ & & & & $\mathrm{NZ}$ & $\mathrm{N}$ & 2004-2005 \\
\hline Alcantara-Moral et al., 2014 & & $31 \%^{c}$ & $58 \%$ & $70 \%$ & Europe & $\mathrm{n} / \mathrm{s}$ & $2009-2013$ \\
\hline Hui et al., 2013 & & $34 \%$ & & & USA & $\mathrm{n} / \mathrm{s}$ & 2005 \\
\hline Hubner et al., 2015 & & $10 \%$ & & & Europe & $\mathrm{n} / \mathrm{s}$ & 2010 \\
\hline Rettenmaier et al., 2014 [DS1] & & & & $38 \%$ & USA & $\mathrm{n} / \mathrm{s}$ & $2009-2013$ \\
\hline Rettenmaier et al., 2014 [DS2] & & & & $37 \%$ & USA & $\mathrm{n} / \mathrm{s}$ & $2009-2013$ \\
\hline Rettenmaier et al., 2014 [DS3] & & & & $32 \%$ & USA & $\mathrm{n} / \mathrm{s}$ & 2009-2013 \\
\hline Rettenmaier et al., 2014 [DS4] & & & & $24 \%$ & Europe & $\mathrm{n} / \mathrm{s}$ & 2010 \\
\hline \multicolumn{8}{|l|}{ Upper gastrointestinal } \\
\hline Garth et al., 2010 [DS2] & $3.6 \pm 2.2 d$ & & & & $\mathrm{AU}$ & $\mathrm{n} / \mathrm{s}$ & $2006-2007$ \\
\hline Selby et al., 2016 & $4(3-5) d^{b}$ & & & & USA & $\mathrm{Y}$ & 2007-2009 \\
\hline Kuwabara et al., 2011 [DS3] & $4.6 \pm 3.7 d$ & & & & Japan & $\mathrm{n} / \mathrm{s}$ & 2007 \\
\hline Kuwabara et al., 2011 [DS1] & $5.3 \pm 3.0 \mathrm{~d}$ & & & & Japan & $\mathrm{n} / \mathrm{s}$ & 2007 \\
\hline Kuwabara et al., 2011 [DS4] & $5.9 \pm 4.2 d$ & & & & Japan & $\mathrm{n} / \mathrm{s}$ & 2007 \\
\hline Kuwabara et al., 2011 [DS2] & $6.7 \pm 5.4 \mathrm{~d}$ & & & & Japan & $\mathrm{n} / \mathrm{s}$ & 2007 \\
\hline Labagga et al., 2016 & & $34 \%$ & & & $\mathrm{AU}$ & $\mathrm{n} / \mathrm{s}$ & 2011 \\
\hline \multicolumn{8}{|l|}{ Obstetric and gynaecological } \\
\hline Benhamou et al., 2002 & & $92 \%$ & $100 \%$ & & Europe & $\mathrm{N}$ & 1996 \\
\hline Conchin et al., 2013 & $1(0-3) d^{b}$ & $0 \%$ & $41 \%$ & & $A U$ & $\mathrm{n} / \mathrm{s}$ & 2010-2011 \\
\hline de Groot et al., 2014 & $2(2-3) d^{b}$ & $0 \%$ & & & Europe & Y & 2007-2008 \\
\hline Deniau et al., 2016 & & $31 \%^{c}$ & & & Europe & $\mathrm{n} / \mathrm{s}$ & $2012-2013$ \\
\hline \multicolumn{8}{|l|}{ Orthopaedic } \\
\hline Christelis et al., 2015 & $3.2(2-5) h^{b}$ & & & & $\mathrm{AU}$ & $\mathrm{n} / \mathrm{s}$ & 2012 \\
\hline Imbelloni et al., 2014 & $17 \pm 2 \mathrm{~h}$ & & & & Brazil & $\mathrm{N}$ & 2001 \\
\hline \multicolumn{8}{|l|}{ Abdominal } \\
\hline Cestonaro et al., 2014 & $15.7(1.7-90.4) \mathrm{h}^{\mathrm{a}}$ & & & & Brazil & $\mathrm{n} / \mathrm{s}$ & 2012 \\
\hline Thompson et al., 2012 & $16(6-28) h^{b}$ & & & & $\mathrm{AU}$ & $\mathrm{n} / \mathrm{s}$ & 2012 \\
\hline Francisco et al., 2015 & $17.4(2.5-29.6) \mathrm{h}^{\mathrm{a}}$ & $25 \%$ & $77 \%$ & $89 \%$ & Brazil & $\mathrm{Y}$ & 2014 \\
\hline De aguilar-Nascimento et al., 2010 & $1(0-10 d) d^{a}$ & & & & Brazil & $\mathrm{N}$ & 2004-2005 \\
\hline De aguilar-Nascimento et al., 2008 & $1(0-20) d^{a}$ & & & & Brazil & $\mathrm{N}$ & 2005 \\
\hline
\end{tabular}

[AU: Australia; DS: data sets; EBG: evidenced based guidelines; D: day; DC: data collection; h: hour; N: no; n/s: not specified; NZ: New Zealand; USA: United States of America; Y: yes]

Note: shading represents practices in-line with evidenced-based feeding recommendations

a Median(range)

${ }^{\mathrm{b}}$ Median(IQR)

'Feed commenced $\leq 12 \mathrm{~h}$ D0

\subsection{Description of feeding practices}

Feeding practices reported by included investigations are described in Table 2. Twelve (41\%) investigations reported data on both first feed and solid feed, while the remaining exclusively reported first feed $(n=10,34 \%)$ or first solid feed $(n=7,24 \%)$ data. Studies reporting on first feed ( $n=22,76 \%)$, presented data as either mean/median time (hours or days) to first feed ( $n=10,45 \%)$, proportion of patients feeding per postoperative day $(n=7,32 \%)$, or both $(n=5$, 
23\%). Similarly, studies reporting first solid feed ( $n=19,66 \%)$, presented data as either mean/median time (hours or days) to first solid feed $(n=7,37 \%)$, proportion of patients feeding on solids per postoperative day $(n=8,42 \%)$, or both $(n=4,21 \%)$. Studies reporting data in the form of proportion of patients feeding postoperatively $(n=16)$, varied in respect to reporting period (i.e. exclusively for D0/D1 or over a period of postoperative days, ranging from D0/D1-D2/D3/D4/D5).

\subsubsection{First feed data and relationship with evidenced-based guidelines}

First feed data and its relationship with evidenced-based guidelines are described in Table 3. Mean/median time to first feed varied with the earliest feed reported within 3.2 hours of surgery and the latest 6.7 days following surgery. In addition, proportional time to first feed data varied. Only three studies reported data until the majority of patients had commenced feeding (i.e. $\geq 75 \%$ : range, D0-D1-D2). Ten (40\%) investigations reported time to first feed in line with evidenced-based recommendations.

The majority of studies commenced oral feeding exclusively via the oral route $(n=17,77 \%)$, while for the remaining, patients either commenced feeding via the oral or enteral route $(n=2)$ or studies did not specify the gastrointestinal route undertaken $(n=3)$ (Table 2). Of the studies which specified the type of diet first commenced $(n=14)$, patients were commenced on fluid only diets in 12 (86\%) and solid diets in two (14\%) investigations.

\subsubsection{First solid feed data and relationship with evidenced-based guidelines}

First solid feed data and its relationship with evidenced-based guidelines are described in Table 4. Mean/median time to first solid feed varied with the earliest commenced within seven hours of surgery and the latest nine days following surgery. In addition, proportional time to first solid feed data varied. The majority of investigations reported $\leq 25 \%$ (range, 0 $5 \%)$ of patients had commenced solid feeding by D1 ( $n=5,50 \%)$. Five studies specified feeding practices until D4 or D5, of which 4 reported $<75 \%$ (range, 40-70\%) of patients were feeding on solids (Table 2). Only three studies reported data until the majority of patients had commenced solid feeding (i.e. $\geq 75 \%$ : range, D0-D1-D4) (Table 2). Four (22\%) investigations reported time to first solid feed in-line with evidenced-based recommendations.

Of the studies which specified the type of first solid feed commenced ( $n=9,47 \%$ ), the majority reported regular diets $(n=5,56 \%)$ (Table 2). Four studies reported the use of light 
$(n=1)$, postoperative $(n=2)$ or various therapeutic $(n=1)$ diets prior to commencement of a regular diet.

Table 4. First solid feed tabulated according to procedural location

\begin{tabular}{|c|c|c|c|c|c|c|c|}
\hline \multirow[t]{2}{*}{ Study } & \multirow{2}{*}{$\begin{array}{l}\text { Mean } \pm S D \text { or } \\
\text { median (IQR) time } \\
\text { to first feed }\end{array}$} & \multicolumn{3}{|c|}{ Proportion } & \multicolumn{3}{|l|}{ Factors } \\
\hline & & Do & D1 & D2 & Origin & EBG & $\mathrm{DC}$ date/s \\
\hline \multicolumn{8}{|c|}{ Lower gastrointestinal (and small intestine) } \\
\hline Schwarzbach et al., 2011 & & & $50 \%$ & $59 \%$ & Europe & $\mathrm{Y}$ & $2006-2007$ \\
\hline Kolozsvari et al., 2013 & $3(2-3.5) d^{a}$ & & & & Canada & Y & 2003-2004 \\
\hline Hardt et al., 2013 & $3.2 \pm 2.7 \mathrm{~d}$ & & $29 \%$ & $52 \%$ & Europe & Y & 2006-2007 \\
\hline Rohatiner et al., 2012 & & $4 \%$ & $4 \%$ & $44 \%$ & USA & $\mathrm{n} / \mathrm{s}$ & 2011 \\
\hline Alcantara-Moral et al., 2014 & & $0 \%$ & $5 \%$ & $40 \%$ & Europe & $\mathrm{Y}$ & 2011 \\
\hline Maessen et al., 2009 & $5 d^{a}$ & $0 \%$ & $3 \%$ & $7 \%$ & Europe & $\mathrm{n} / \mathrm{s}$ & 2004 \\
\hline \multicolumn{8}{|l|}{ Upper gastrointestinal } \\
\hline Selby et al., 2016 & $6(5-7) d^{a}$ & & & & USA & $\mathrm{n} / \mathrm{s}$ & $2007-2009$ \\
\hline Hilal et al., 2013 & $9(6-12) d^{a}$ & & & & Europe & $\mathrm{N}$ & $2009-2010$ \\
\hline Labagga et al., 2016 & & & $5 \%$ & & $\mathrm{AU}$ & $\mathrm{n} / \mathrm{s}$ & 2011 \\
\hline \multicolumn{8}{|l|}{ Obstetric and gynaecological } \\
\hline Benhamou et al., 2002 & & $92 \%$ & $100 \%$ & & Europe & $\mathrm{N}$ & 1996 \\
\hline de Groot et al., 2014 & $3(3-4) d^{a}$ & $0 \%$ & & & Europe & Y & $2007-2008$ \\
\hline Deniau et al., 2016 & & $20 \%{ }^{c}$ & & & Europe & $\mathrm{n} / \mathrm{s}$ & $2012-2013$ \\
\hline Conchin et al., 2013 & $4(3-6) d^{a}$ & $0 \%$ & $0 \%$ & $13 \%$ & $\mathrm{AU}$ & $\mathrm{n} / \mathrm{s}$ & $2010-2011$ \\
\hline Kalogera et al., 2013 & $5(3-7) d^{a}$ & & & & USA & $\mathrm{n} / \mathrm{s}$ & 2010 \\
\hline \multicolumn{8}{|l|}{ Orthopaedic } \\
\hline Christelis et al., 2015 & $7(4.3-15) h^{a}$ & & & & $\mathrm{AU}$ & $\mathrm{n} / \mathrm{s}$ & 2012 \\
\hline Imbelloni et al., 2014 & $17 \pm 2 h$ & & & & Brazil & $\mathrm{N}$ & 2001 \\
\hline Egerod et al., 2010 & & & $66 \%$ & & Europe & $\mathrm{Y}$ & 2007-2008 \\
\hline \multicolumn{8}{|l|}{ Abdominal } \\
\hline Francisco et al., 2015 & $18.3(3.3-98.8) \mathrm{h}^{b}$ & $23 \%$ & $77 \%$ & $89 \%$ & Brazil & $Y$ & 2014 \\
\hline Thompson et al., 2012 & $2.8(1.1-5) d^{a}$ & & & & $\mathrm{AU}$ & $\mathrm{n} / \mathrm{s}$ & 2009 \\
\hline
\end{tabular}

[AU: Australia; DS: data sets; EBG: evidenced based guidelines; D: day; DC: data collection; h: hour; N: no; n/s: not specified; USA: United States of America; Y: yes]

Note: shading represents practices in-line with evidenced-based feeding recommendations

${ }^{a}$ Median(IQR)

${ }^{b}$ Median(range)

${ }^{\mathrm{c}}$ Feed commenced $\leq 12 \mathrm{~h}$ D0

\subsubsection{First feed and first solid feed data by postoperative patient group}

First feed and first solid feed data by postoperative patient group are depicted in Tables 3 and 4, respectively. The shortest median/mean times to first feed and first solid feed were observed in orthopaedic investigations (3-17 hours and 7-17 hours, respectively) and the longest in lower (range, 1-2 days and 3-5 days, respectively) and upper (range, 3.6-6.7 days and 6-9 days, respectively) GI investigations. One (6\%), zero (0\%), three (33\%), four (80\%) and six (86\%) lower GI, upper GI, obstetric and gynaecological, orthopaedic and abdominal data sets, reported liquid and/or solid feeding practices in-line with evidenced-based recommendations, respectively.

\subsubsection{Patient-related data}

Only five studies (17\%) reported data on the prevalence of postoperative nausea and/or vomiting (PONV); factors which may directly impact on oral/enteral intake ${ }^{(48,51,63,71,72)}$. 
Two studies reported PONV prevalence in the context of postprandial feeding (i.e. following feeding); ranging from $23 \%{ }^{(71)}$ to $39 \%{ }^{(48)}$. Of the remaining studies two reported low rates of perceived postoperative nausea (6\% and a median score of one out of 10$)^{(51,72)}$ and one reported higher prevalence's of postoperative nausea (45\%) and vomiting (26\%) ${ }^{(63)}$ in the postoperative period. Few studies $(n=3)$ reported perceived thirst (range, 63-74\%) and hunger (range, 38-60\%) prevalence among postoperative patients (D0-D1) ${ }^{(51,52,59)}$.

\subsection{Quality assessment}

Quality assessment scores of included studies are summarised in Table S1 (Supplementary Information). Overall, 20 studies (69\%) scored above 70\% (range, 38\% - 89\%) on quality assessment. The majority of studies addressed a clearly focused question $(n=29,100 \%)$, defined criteria for inclusion/exclusion $(n=23,79 \%)$ and used appropriate statistical tests $(n=29,100 \%)$. Few studies measured outcomes in a valid and reliable way ( $n=14,48 \%)$ and collected data long enough to be meaningful ( $n=17,58 \%)$, respectively. 


\section{Discussion ${ }^{(67)}$}

The aim of this study was to investigate postoperative feeding practices among hospitalised patients. Although early postoperative feeding is a strategy that can contribute to improved recovery and reduced complications after surgery ${ }^{(1,5,6,9-18)}$, it does not appear to be commonly applied in clinical practice. Of the 29 studies meeting the inclusion criteria, only $40 \%$ and $22 \%$ reported time to first feed and time to first solid feed in line with evidencedbased recommendations, respectively. Further, patients who underwent lower or upper GI surgeries appeared more likely to experience delayed feeding. These findings demonstrate limited translation of the evidence for early post-operative feeding into routine practice, and the need for ongoing interventions targeted at improving postoperative nutrition delivery.

Less than half of included investigations commenced feeding within 24 hours of surgery and of these, the majority reported liquids as first oral intake (86\%). Liquids, in contrast to solid foods, mitigate gastrointestinal and pancreatic secretion, providing a theoretical advantage in avoiding the development of paralytic ileus ${ }^{(74)}$; however, given they are nutritionally inadequate, prolonged use is not advised ${ }^{(75)}$. Prompt prescription of solid diets (i.e. within 24 hours of surgery) that have the potential to deliver adequate nutrition is therefore recommended to prevent malnutrition ${ }^{(76)}$. A rapid transition to solid foods may also increase patient satisfaction (e.g. improved sense of well-being and lower pain perceptions) and decrease length of stay and number of days to first flatus ${ }^{(77,78)}$. Despite this, over half of investigations reported a median time of three or more days (up to nine days) to commence solid feeding. Further, when solid diets were initiated, 44\% of investigations reported using 'special/therapeutic' diets (i.e. low fibre/residual, cardiac, postoperative, light, soft), which are often restrictive ${ }^{(76)}$, nutritionally inadequate ${ }^{(79)}$ and have little scientific purpose in the context of postoperative care ${ }^{(76)}$. Collectively, these findings indicate a gap between evidenced-based recommendations and practice for initiating and progressing patients onto nutritionally adequate solid (i.e. regular) diets following surgery. A number of factors may contribute to this finding, including the surgical procedure undertaken, patient and/or health care staff knowledge/attitudes/beliefs, the existence and implementation of evidenced-based guidelines/protocols and limitations of the hospital and foodservice systems.

The surgical population investigated appeared to influence whether investigations reported feeding practices in-line with evidence-based recommendations. Patients who underwent orthopaedic surgeries commenced liquid and solid feeding typically earlier and were more 
likely to begin feeding within 24 hours of surgery compared to patients who underwent GI procedures. However, poor feeding practices were not observed among all GI investigations. The majority of patients who underwent unspecified 'abdominal' procedures commenced liquid and solid feeding within 24 hours of surgery compared to those who underwent specified upper and lower GI procedures. Differences in procedural complexity may account for this, considering the majority of 'abdominal' investigations had patients which underwent less complex procedures (e.g. hernioplasty, cholecystectomy) in comparison to specified GI investigations where patients underwent more complex lower (e.g. hemicolectomy, sigmoidectomy) or upper (e.g. gastrectomy, pancreatectomy) GI surgeries. Previous studies have also identified procedural complexity (i.e. length of surgery, 'minor' vs 'major' or 'partial' vs 'complete' procedures), in addition to surgical location (e.g. upper vs lower GI), as features influencing time to commence/progress postoperative feeding $(47,54,55)$. Given postoperative care guidelines take into account procedural location and complexity, why these factors continue to influence time to commence liquid and solid feeding remains unclear; however, historic dictums may be a contributing factor. While traditional feeding protocols (i.e. nothing-by-mouth following surgery until clinical signs of bowel function return) originated from fears early feeding could result in severe paralytic ileus, wound dehiscence and anastomotic leakage ${ }^{(1)}$; outcomes more likely to occur following 'complex' GI resection surgery, the extent to which these beliefs may still influence current practice has received limited scientific attention ${ }^{(80)}$. Nonetheless, the prevalence of delayed feeding among patient populations arguably in the greatest need of adequate nutrition following surgery (upper and lower GI patients) is concerning.

Patient-related factors (e.g. PONV, fear, food preferences/options) may also influence when postoperative feeding is initiated. For example, patients may self-delay commencing and progressing postoperative feeding due to or in fear of PONV ${ }^{(81)}$. Equivocal results were observed among investigations reporting PONV prevalence's (6-45\%). Previous studies suggest PONV is a leading cause of patient dissatisfaction, with patients reporting they would prefer to suffer from pain rather than PONV ${ }^{(82)}$ and are willing to pay a considerable sum of money for an effective anti-emetic ${ }^{(83)}$. Alternatively, patients may feel hungry or thirsty following surgery ${ }^{(51,52,59)}$; however, limited or unpalatable dietary options (e.g. the clear liquid diet) may prevent patients from commencing feeding ${ }^{(84)}$. A patient-centred approach where patients receive effective anti-emetics and timely prescription of unrestricted diets (i.e. 'regular diets') may lead to earlier commencement of feeding. Lastly, given females are at 
greater risk of PONV ${ }^{(85)}$, and elderly individuals experience reduced appetites ${ }^{(86)}$, future investigations should consider whether postoperative feeding practices are influenced by age and gender.

Foodservice-related factors (e.g. dietary options and meal delivery) may contribute to when patients can initiate postoperative feeding. Common food options preferred among postoperative patients (within 24 hours of surgery) include, toast, fresh fruit, potatoes, eggs, crackers, pudding, and sandwiches ${ }^{(84)}$. Given the majority of these foods are not offered by hospitals to patients on liquid or 'special' diets; diet types commonly prescribed after surgery, may in part explain the poor feeding practices observed in the current review. Further, a delay in the delivery of meals to patients following diet prescription may also contribute. For example, patients returning from afternoon surgery may miss dinner delivery and consequently receive their first meal the following day. However, while plausible, this is unlikely to explain the substantial deviations from evidence-based guidelines for commencing feeding reported by some studies $(7,47,48,50,56,71,73)$. Given the paucity of evidence on foodservice-related factors and the commencement of early oral feeding, further research is warranted to determine if this is a contributing factor.

The current review also examined feeding practices in the context of the existence and implementation of evidenced-based guidelines/protocols. Neither data collection timeframes (before or after 2006) nor whether hospitals had evidence-based feeding protocols in place during data collection appeared to influence feeding practices. Despite early oral feeding guidelines originating in Europe ${ }^{(32)}$, European investigations did not appear to report more favourable feeding practices. Considering feeding recommendations for surgical groups have been released at different times, it is possible that limited exposure to guideline availability may have influenced findings. However, early oral feeding guidelines were first established in 2006 for colorectal patients ${ }^{(32)}$, yet only 6\% of lower GI data sets (amongst the lowest) reported feeding practices in-line with evidenced-based recommendations. Collectively, these findings suggest that the mere availability of compelling evidence-based guidelines has little effect on influencing habitual practices; a similar finding reported by other studies investigating uptake of evidence-based protocols (e.g. 'Fast Track' and 'Early Recovery After Surgery’ programs) into daily practice ${ }^{(37,38)}$. Previous work has shown barriers to change in clinical practice can arise at different stages in the healthcare system (e.g. at the patient, individual professional, health-care team or health-care organisation level) ${ }^{(36)}$. Further research is therefore required to explore physician (i.e. delayed diet prescription), 
organisational (i.e. delayed foodservice delivery) and patient-related (i.e. delayed diet intake) factors impeding adherence to evidenced-based recommendations, particularly among GI patients, in order to develop interventions to improve postoperative feeding.

Several limitations of the present review are acknowledged. Firstly, inconsistencies with how data were reported (median/mean time to first feed vs number of patients eating per postoperative day) limited our ability to directly compare feeding practices across studies. We attempted to account for this by categorising data sets into binary groups; those 'inline' and those not 'in-line with evidenced-based guidelines'. However, we recognise the two measures may not precisely correlate (e.g. a mean/median time to first feed of 1 day $=75 \%$ of cohort commenced feeding by D1). Further, proportional data is a broad measure (D0 could represent within 24 hours of surgery or the day of surgery e.g. within $\sim 12$ hours), thus in effort to not overestimate the problem, studies $(n=4)(57,62,63,67)$ which reported proportional data exclusively for D0 were not included when the percentage of data sets 'in-line with evidenced-based guidelines' were calculated. While differences in feeding practices across data sets were attributed to surgical location and complexity, other perioperative factors may also contribute. Previous studies have proposed pain management strategies (epidural vs general anaesthetic) and surgical approach (laparotomy vs laparoscopy) ${ }^{(87)}$ can influence time to commence feeding. Due to inconsistencies with reporting these variables and the heterogeneity between studies (different surgical populations), the current review did not investigate whether these factors influenced time to commence feeding. Lastly, feeding practices for certain postoperative groups were represented by 2-4 studies and over half of included studies were deemed 'unclear' in respect to having measured outcomes in a valid and reliable way, thus our findings need to be interpreted with caution. Further high quality prospective research is required to investigate feeding practices across different postoperative groups.

\section{Conclusion}

This review found that the majority of postoperative patients are not fed within timeframes outlined by evidence-based guidelines. While both poor liquid and solid feeding were observed, timely upgrade of patients on to nutritionally adequate diets (i.e. 'regular' diets) appears particularly problematic. Given adherence to evidenced-based feeding recommendations may be reliant on physician (i.e. diet prescription), organisational (i.e. diet delivery) and patient-related (i.e. diet commencement) factors, further research exploring 
these parameters, particularly among upper and lower GI patients, is warranted. This information will help inform strategies for postoperative nutrition delivery interventions to improve patient and health care-related outcomes.

\section{Transparency Declaration}

The lead author affirms that this manuscript is an honest, accurate, and transparent account of the study being reported. The reporting of this work is compliant with CONSORT ${ }^{1} /$ STROBE $^{2} /$ PRISMA $^{3}$ guidelines. The lead author affirms that no important aspects of the study have been omitted and that any discrepancies from the study as planned (please add in the details of any organisation ${ }^{4}$ that the trial or protocol has been registered with) have been explained.

\section{Conflict of interest and funding disclosure}

The authors declare no conflicts of interests. This study received internal institutional support only.

\section{Acknowledgments}

The authors would like to thank Jenny Campagnolo for her assistance with developing the search strategy. 
Table S1. Methodological quality assessment summary of included studies

\begin{tabular}{|c|c|c|c|c|c|c|c|c|c|c|c|}
\hline Citation & 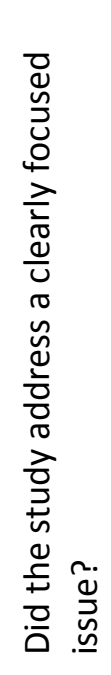 & 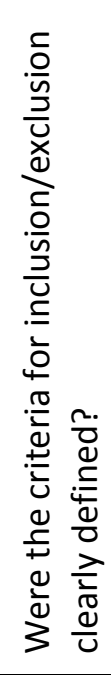 & 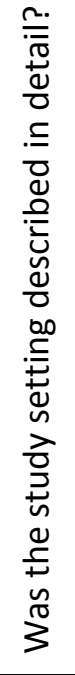 & 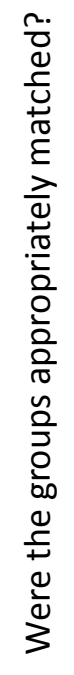 & 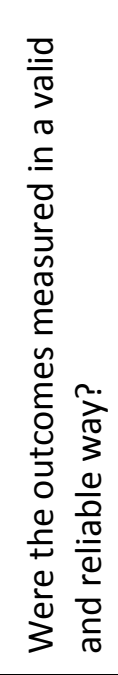 & 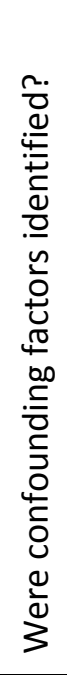 & 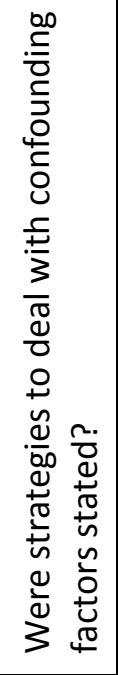 & 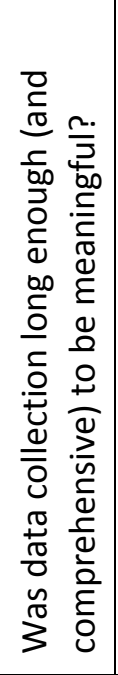 & 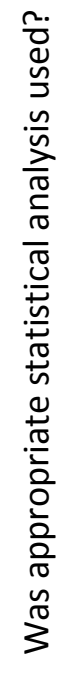 & 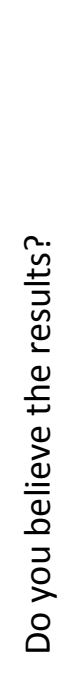 & 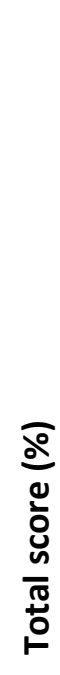 \\
\hline Alcantara-Moral et al., 2014 & $\mathrm{Y}$ & $\mathrm{Y}$ & $\mathrm{Y}$ & $\mathrm{N}$ & $u$ & $\mathrm{Y}$ & $\mathrm{Y}$ & $\mathrm{Y}$ & $\mathrm{y}$ & $u$ & $70 \%$ \\
\hline Benhamou et al., 2002 & $\mathrm{Y}$ & $\mathrm{Y}$ & u & NA & u & u & NA & $\mathrm{Y}$ & $\mathrm{Y}$ & u & $44 \%$ \\
\hline Cestonaro et al., 2014 & $\mathrm{Y}$ & $\mathrm{Y}$ & $\mathrm{Y}$ & NA & u & $\mathrm{Y}$ & $\mathrm{u}$ & $\mathrm{Y}$ & Y & $\mathrm{Y}$ & $78 \%$ \\
\hline Christelis et al., 2015 & $\mathrm{Y}$ & u & $\mathrm{Y}$ & NA & $\mathrm{Y}$ & $\mathrm{Y}$ & NA & $\mathrm{Y}$ & $\mathrm{Y}$ & $\mathrm{Y}$ & $78 \%$ \\
\hline Conchin et al., 2013 & $\mathrm{Y}$ & $\mathrm{Y}$ & $\mathrm{Y}$ & NA & u & $\mathrm{Y}$ & NA & $\mathrm{N}$ & $\mathrm{Y}$ & $\mathrm{Y}$ & $75 \%$ \\
\hline $\begin{array}{l}\text { de Aguilar-Nascimento et al., } \\
2008\end{array}$ & $\mathrm{Y}$ & $u$ & u & N/A & u & $\mathrm{Y}$ & $u$ & $\mathrm{Y}$ & $\mathrm{Y}$ & $\mathrm{u}$ & $44 \%$ \\
\hline $\begin{array}{l}\text { de Aguilar-Nascimento et al., } \\
2010\end{array}$ & $\mathrm{Y}$ & $\mathrm{Y}$ & $\mathrm{Y}$ & N/A & u & $\mathrm{Y}$ & u & $\mathrm{Y}$ & $\mathrm{Y}$ & u & $67 \%$ \\
\hline de Groot et al., 2014 & $\mathrm{Y}$ & $\mathrm{Y}$ & $\mathrm{Y}$ & NA & $\mathrm{Y}$ & $\mathrm{Y}$ & $\mathrm{Y}$ & U & $\mathrm{Y}$ & $\mathrm{Y}$ & $89 \%$ \\
\hline Deniau et al., 2016 & $\mathrm{Y}$ & $\mathrm{Y}$ & $\mathrm{Y}$ & NA & $\mathrm{Y}$ & $\mathrm{Y}$ & NA & $\mathrm{U}$ & $\mathrm{Y}$ & $\mathrm{Y}$ & $88 \%$ \\
\hline Egerod et al., 2010 & $\mathrm{Y}$ & $u$ & $\mathrm{Y}$ & NA & $\mathrm{Y}$ & $\mathrm{Y}$ & NA & $\mathrm{Y}$ & $\mathrm{Y}$ & $\mathrm{Y}$ & $86 \%$ \\
\hline Francisco et al., 2015 & $\mathrm{Y}$ & $\mathrm{Y}$ & u & NA & u & $\mathrm{Y}$ & u & U & $\mathrm{Y}$ & u & $44 \%$ \\
\hline Garth et al., $2010^{\circ}$ & $\mathrm{Y}$ & $\mathrm{Y}$ & $\mathrm{Y}$ & NA & $\mathrm{Y}$ & $\mathrm{Y}$ & u & $\mathrm{Y}$ & $\mathrm{Y}$ & $\mathrm{Y}$ & $89 \%$ \\
\hline Hardt et al., $2013^{\mathrm{c}}$ & $\mathrm{Y}$ & $\mathrm{Y}$ & u & NA & u & $\mathrm{Y}$ & $\mathrm{Y}$ & u & $\mathrm{Y}$ & $\mathrm{Y}$ & $67 \%$ \\
\hline Hilal et al., 2013 & $\mathrm{Y}$ & $\mathrm{Y}$ & $\mathrm{Y}$ & NA & $u$ & $\mathrm{Y}$ & NA & u & $\mathrm{Y}$ & $\mathrm{Y}$ & $75 \%$ \\
\hline Hubner et al., 2015 & $\mathrm{Y}$ & $\mathrm{Y}$ & u & NA & $\mathrm{Y}$ & $\mathrm{Y}$ & NA & U & $\mathrm{Y}$ & $\mathrm{Y}$ & $75 \%$ \\
\hline Hui et al., 2013 & $\mathrm{Y}$ & $\mathrm{Y}$ & $\mathrm{Y}$ & NA & u & $\mathrm{Y}$ & NA & $\mathrm{Y}$ & $\mathrm{Y}$ & $\mathrm{Y}$ & $88 \%$ \\
\hline Imbelloni et al., 2014 & $\mathrm{Y}$ & $\mathrm{Y}$ & u & NA & u & $\mathrm{Y}$ & NA & $\mathrm{Y}$ & $\mathrm{Y}$ & $\mathrm{Y}$ & $75 \%$ \\
\hline Kalogera et al., 2013 & $\mathrm{Y}$ & $\mathrm{Y}$ & $\mathrm{Y}$ & NA & u & $\mathrm{Y}$ & NA & $\mathrm{Y}$ & $\mathrm{Y}$ & $\mathrm{Y}$ & $88 \%$ \\
\hline Kolozsvari et al., 2013 & $\mathrm{Y}$ & $\mathrm{Y}$ & $\mathrm{Y}$ & NA & u & $\mathrm{Y}$ & NA & $\mathrm{Y}$ & $\mathrm{Y}$ & $\mathrm{Y}$ & $88 \%$ \\
\hline Kuwabara et al., 2011 & $\mathrm{Y}$ & $\mathrm{N}$ & $\mathrm{Y}$ & NA & $\mathrm{Y}$ & $\mathrm{Y}$ & $\mathrm{Y}$ & $\mathrm{Y}$ & $\mathrm{Y}$ & $\mathrm{Y}$ & $89 \%$ \\
\hline Labgaa et al., 2016 & $\mathrm{Y}$ & $\mathrm{Y}$ & u & NA & $\mathrm{Y}$ & $\mathrm{Y}$ & NA & U & $\mathrm{Y}$ & $\mathrm{Y}$ & $75 \%$ \\
\hline Maessen et al., 2009 & $\mathrm{Y}$ & $u$ & $\mathrm{Y}$ & NA & $\mathrm{Y}$ & $\mathrm{Y}$ & $\mathrm{Y}$ & $\mathrm{Y}$ & $\mathrm{Y}$ & $\mathrm{Y}$ & $89 \%$ \\
\hline Miller et al., 2014 & $\mathrm{Y}$ & $u$ & u & NA & $u$ & $\mathrm{Y}$ & NA & U & $\mathrm{Y}$ & U & $38 \%$ \\
\hline Rettenmaier et al., 2014 & $\mathrm{Y}$ & $\mathrm{Y}$ & $u$ & NA & $\mathrm{u}$ & $\mathrm{Y}$ & $\mathrm{Y}$ & $\mathrm{Y}$ & $\mathrm{Y}$ & $\mathrm{Y}$ & $78 \%$ \\
\hline
\end{tabular}




\begin{tabular}{|c|c|c|c|c|c|c|c|c|c|c|c|}
\hline Rohatiner et al., $2012^{\mathrm{c}}$ & $\mathrm{Y}$ & $\mathrm{Y}$ & $\mathrm{Y}$ & NA & $\mathrm{Y}$ & $\mathrm{Y}$ & $\mathrm{Y}$ & u & $\mathrm{Y}$ & $\mathrm{Y}$ & $89 \%$ \\
\hline Schwarzbach et al., 2011 & $\mathrm{Y}$ & $\mathrm{Y}$ & $\mathrm{u}$ & NA & $\mathrm{U}$ & $\mathrm{Y}$ & NA & $\mathrm{Y}$ & $\mathrm{Y}$ & $\mathrm{Y}$ & $75 \%$ \\
\hline Selby et al., 2016 & $\mathrm{Y}$ & $\mathrm{Y}$ & $\mathrm{u}$ & NA & $\mathrm{u}$ & $\mathrm{Y}$ & $\mathrm{Y}$ & $\mathrm{u}$ & $\mathrm{Y}$ & $\mathrm{u}$ & $56 \%$ \\
\hline Thompson et al., 2012 & $\mathrm{Y}$ & $\mathrm{Y}$ & $\mathrm{U}$ & NA & $\mathrm{Y}$ & $\mathrm{Y}$ & NA & $\mathrm{Y}$ & $\mathrm{Y}$ & $\mathrm{Y}$ & $89 \%$ \\
\hline Zargar-Shoshtari et al., 2008 & Y & $\mathrm{Y}$ & U & NA & $\mathrm{U}$ & $\mathrm{Y}$ & $\mathrm{u}$ & $\mathrm{U}$ & $\mathrm{Y}$ & $\mathrm{U}$ & $44 \%$ \\
\hline
\end{tabular}




\section{References}

1. Bisgaard $\mathrm{T}$, Kehlet $\mathrm{H}$. Early oral feeding after elective abdominal surgery--what are the issues? Nutrition 2002; 18: 944-8.

2. Nelson G, Altman AD, Nick A, Meyer LA, Ramirez PT, Achtari C et al. Guidelines for postoperative care in gynecologic/oncology surgery: Enhanced Recovery After Surgery (ERAS ${ }^{\circledR}$ ) Society recommendations - Part II. Gynecol Oncol 2016; 140: 323-32.

3. Fanning J, Hojat R. Safety and efficacy of immediate postoperative feeding and bowel stimulation to prevent ileus after major gynecologic surgical procedures. J Am Osteopath Assoc 2011; 111: 469-72.

4. Andersen HK, Lewis SJ, Thomas S. Early enteral nutrition within $24 \mathrm{~h}$ of colorectal surgery versus later commencement of feeding for postoperative complications. Cochrane Database of Syst Rev 2006; 1.

5. Charoenkwan K, Matovinovic E. Early versus delayed oral fluids and food for reducing complications after major abdominal gynaecologic surgery. Cochrane Database of Syst Rev 2014; 12.

6. Fujii T, Morita H, Sutoh T, Yajima R, Yamaguchi S, Tsutsumi S et al. Benefit of oral feeding as early as one day after elective surgery for colorectal cancer: oral feeding on first versus second postoperative day. Int Surg 2014; 99: 211-5.

7. Garth A, Newsome C, Simmance N, Crowe T. Nutritional status, nutrition practices and postoperative complications in patients with gastrointestinal cancer. J Hum Nutr Diet 2010; 23: 393-401.

8. Fettes S, Davidson I, Richardson R, Pennington C. Nutritional status of elective gastrointestinal surgery patients pre- and post-operatively. Clin Nutr 2002; 21: 249-54.

9. Pragatheeswarane M, Muthukumarassamy R, Kadambari D, Kate V. Early Oral Feeding vs. Traditional Feeding in Patients Undergoing Elective Open Bowel Surgery-a Randomized Controlled Trial. J Gastrointest Surg 2014; 18: 1017-23.

10. Hosseini SN, Mousavinasab SN, Rahmanpour H, Sotodeh S. Comparing early oral feeding with traditional oral feeding in upper gastrointestinal surgery. Turk J Gastroenterol 2010; 21: 119-24. 11. Hwang $\mathrm{S}$, Jung $\mathrm{M}, \mathrm{Cho} B, \mathrm{Yu} \mathrm{H}$. Clinical feasibility and nutritional effects of early oral feeding after pancreaticoduodenectomy. Korean J Hepatobiliary Pancreat Surg 2014; 18: 84-9.

12. Minig L, Biffi R, Zanagnolo V, Attanasio A, Beltrami C, Bocciolone L et al. Reduction of postoperative complication rate with the use of early oral feeding in gynecologic oncologic patients undergoing a Major surgery: A randomized controlled trial. Ann Surg Oncol 2009; 16: 3101-10.

13. Lewis $S$, Egger $M$, Sylvester $P$, Thomas $S$. Early enteral feeding versus "nil by mouth" after gastrointestinal surgery: systematic review and meta-analysis of controlled trials. J Gastrointest Surg 2001; 323: 773-6.

14. Lewis S, Andersen H, Thomas S. Early Enteral Nutrition Within $24 \mathrm{~h}$ of Intestinal Surgery Versus Later Commencement of Feeding: A Systematic review and Meta-analysis. J Gastrointest Surg 2009; 13: 569-75.

15. Zhuang C, Ye X, Zhang C, Dong Q, Chen B, Yu Z. Early versus traditional postoperative oral feeding in patients undergoing elective colorectal surgery: a meta-analysis of randomized clinical trials. Digest surg 2013; 30: 225-32.

16. Nantasupha C, Ruengkhachorn I, Ruangvutilert P. Effect of conventional diet schedule, early feeding and early feeding plus domperidone on postcesarean diet tolerance: A randomized controlled trial. J Obstet Gynaecol Res 2016; 42: 519-25.

17. Masood S, Masood Y, Naim U, Masood M. A randomized comparative trial of early initiation of oral maternal feeding versus conventional oral feeding after cesarean delivery. Int J Gynaecol Obstet 2014; 126: 115-9.

18. Klappenbach RF, Yazyi FJ, Alonso Quintas F, Horna ME, Alvarez Rodríguez J, Oría A. Early Oral Feeding Versus Traditional Postoperative Care After Abdominal Emergency Surgery: A Randomized Controlled Trial. World J Surg 2013; 37: 2293-9.

19. Guo J, Long S, Li H, Luo J, Han D, He T. Early versus delayed oral feeding for patients after cesarean. Int J Gynaecol Obstet 2015; 128: 100-5. 
20. Hsu Y, Hung H, Chang S, Chang Y. Early oral intake and gastrointestinal function after cesarean delivery: A Systematic Review and Meta-Analysis. Obstet Gynecol 2013; 121: 1327-34.

21. Willcutts K, Chung M, Erenberg C, Finn K, Schirmer B, Byham-Gray L. Early Oral Feeding as Compared With Traditional Timing of Oral Feeding After Upper Gastrointestinal Surgery: A Systematic Review and Meta-analysis. Ann surg 2016; 264: 54-63.

22. Osland E, Yunus R, Khan S, Memon M. Early Versus Traditional Postoperative Feeding in Patients Undergoing Resectional Gastrointestinal Surgery: A Meta-Analysis. JPEN 2011; 35: 473-87. 23. Schaible UE, Kaufmann SH. Malnutrition and infection: complex mechanisms and global impacts. PLoS Med 2007; 4: e115.

24. Vivanti A, Ward N, Haines T. Nutritional status and associations with falls, balance, mobility and functionality during hospital admission. J Nutr Health Aging 2011; 15: 388-91.

25. Neyens J, Halfens R, Spreeuwenberg M, Meijers J, Luiking $\mathrm{Y}$, Verlaan $\mathrm{G}$ et al. Malnutrition is associated with an increased risk of falls and impaired activity in elderly patients in Dutch residential long-term care (LTC): a cross-sectional study. Arch Gerontol Geriatr 2013; 56: 265-9.

26. Banks M, Bauer J, Graves N, Ash S. Malnutrition and pressure ulcer risk in adults in Australian health care facilities. Nutrition 2010; 26: 896-901.

27. Lim S, Ong K, Chan Y, Loke W, Ferguson M, Daniels L. Malnutrition and its impact on cost of hospitalization, length of stay, readmission and 3-year mortality. Clin Nutr 2012; 31: 345-50.

28. Jeong O, Ryu SY, Jung MR, Choi WW, Park YK. The safety and feasibility of early postoperative oral nutrition on the first postoperative day after gastrectomy for gastric carcinoma. Gastric Cancer 2014; 17: 324-31.

29. Plauth M, Cabré E, Riggio O, Assis-Camilo M, Pirlich M, Kondrup J et al. ESPEN Guidelines on Enteral Nutrition: Liver disease. Clin Nutr 2006; 25: 285-94.

30. Lassen K, Coolsen MME, Slim K, Carli F, de Aguilar-Nascimento JE, Schäfer M et al. Guidelines for Perioperative Care for Pancreaticoduodenectomy: Enhanced Recovery After Surgery (ERAS ${ }^{\circledR}$ ) Society Recommendations. World J Surg 2013; 37: 240-58.

31. Mortensen K, Nilsson M, Slim K, Schäfer M, Mariette C, Braga M et al. Consensus guidelines for enhanced recovery after gastrectomy. Br J Surg 2014; 101: 1209-29.

32. Weimann A, Braga M, Harsanyi L, Laviano A, Ljungqvist O, Soeters $P$ et al. ESPEN Guidelines on Enteral Nutrition: Surgery including Organ Transplantation. Clin Nutr 2006; 25: 224-44.

33. Nygren J, Thacker J, Carli F, Fearon KCH, Norderval S, Lobo DN et al. Guidelines for Perioperative Care in Elective Rectal/Pelvic Surgery: Enhanced Recovery After Surgery (ERAS ${ }^{\circledR}$ ) Society Recommendations. World J Surg 2013; 37: 285-305.

34. Gustafsson UO, Scott MJ, Schwenk W, Demartines N, Roulin D, Francis N et al. Guidelines for Perioperative Care in Elective Colonic Surgery: Enhanced Recovery After Surgery (ERAS ${ }^{\circledR}$ ) Society Recommendations. World J Surg 2013; 37: 259-84.

35. Graham I, Logan J, Harrison M, Strauss S, Tetroe J, Caswell W et al. Lost in knowledge translation: time for a map? J Contin Educ Health Prof 2006; 26: 13-24.

36. Grol R, Grimshaw J. From best evidence to best practice: effective implementation of change in patients' care. Lancet 2003; 362: 1225-30.

37. Zonca P, Stigler J, Maly T, Neoral C, Hajek M, Stiglerova S. Do we really apply fast-track surgery? Bratis/ Lek Listy 2008; 109: 61-5.

38. Kehlet H, Buchler MW, Beart RW, Jr., Billingham RP, Williamson R. Care after colonic operation--is it evidence-based? Results from a multinational survey in Europe and the United States. J Am Coll Surg 2006; 202: 45-54.

39. Wiltsey Stirman S, Kimberly J, Cook N, Calloway A, Castro F, Charns M. The sustainability of new programs and innovations: a review of the empirical literature and recommendations for future research. Implement Sci 2012; 7: 17.

40. Stroup DF, Berlin JA, Morton SC, Olkin I, Williamson GD, Rennie D et al. Meta-analysis of Observational Studies in Epidemiology: A Proposal for Reporting. JAMA 2000; 283: 2008-12. 
41. Trelle S. Information management and reading habits of German diabetologists: a questionnaire survey. Diabetologia 2002; 45.

42. Franklin GA, McClave SA, Hurt RT, Lowen CC, Stout AE, Stogner LL et al. Physician-Delivered Malnutrition: Why Do Patients Receive Nothing by Mouth or a Clear Liquid Diet in a University Hospital Setting? JPEN 2011; 35: 337-42.

43. Conchin S, Muirhead R, Ferrie S, Carey S. Can't we just let them eat?: Defining and addressing under-use of the oral route in a post-surgical ward. Asia Pac J Clin Nutr 2013; 22: 200-5. 44. The Jonna Briggs Institute. Critical Appraisal Tools 2016. Available from: http://joannabriggs.org/research/critical-appraisal-tools.html.

45. Critical Apprasial Skills Programme. CASP Checklists 2013. Available from: http://www.caspuk.net/checklists.

46. American Society of Anesthesiologists. ASA Physical Status Classification System 2016. Available from: https://www.asahq.org/resources/clinical-information/asa-physical-statusclassification-system.

47. Kuwabara K, Matsuda S, Ishikawa K, Horiguchi H, Fujimori K. Association of Operating Time and Gastrectomy with Initiation of Postoperative Oral Food Intake. Dig Surg 2011; 28: 157-62.

48. Rettenmaier M, Abaid L, Brown J, Mendivil A, Micha J, Goldstein B. The incidence of postprandial nausea and nutritional regression in gynecologic cancer patients following intestinal surgery: A retrospective cohort study. Int J Surg 2014; 12: 783-7.

49. Rohatiner T, Wend J, Rhodes S, Murrell Z, Berel D, Fleshner P. A prospective singleinstitution evaluation of current practices of early postoperative feeding after elective intestinal surgery. Am Surg 2012; 78: 1147-50.

50. Alcántara-Moral M, Serra-Aracil X, Gil-Egea MJ, Frasson M, Flor-Lorente B, Garcia-Granero E et al. Observational cross-sectional study of compliance with the fast track protocol in elective surgery for colon cancer in Spain. Int J Colorectal Dis 2014; 29: 477-83.

51. Benhamou D, Técsy M, Parry N, Mercier FJ, Burg C. Audit of an early feeding program after Cesarean delivery: Patient wellbeing is increased. Can J Anaesth 2002; 49: 814-9.

52. Cestonaro T, Schieferdecker MEM, Thieme RD, Cardoso JN, Campos ACL. The reality of the surgical fasting time in the era of the ERAS protocol. Nutr Hosp 2014; 29: 437-43.

53. Christelis N, Wallace S, Sage CE, Babitu U, Liew S, Dugal J et al. An enhanced recovery after surgery program for hip and knee arthroplasty. Med J Aust 2015; 202: 363-8.

54. de Aguilar-Nascimento J, Bicudo-Salomão A, Caporossi C, Silva R, Cardoso E, Santos T.

Enhancing surgical recovery in Central-West Brazil: The ACERTO protocol results. e-SPEN 2008; 3: 78-

83.

55. de Aguilar-Nascimento JEd, Salomão AB, Caporossi C, Diniz BN. Clinical benefits after the implementation of a multimodal perioperative protocol in elderly patients. Arq Gastroenterol 2010; 47: 178-83.

56. de Groot J, van Es L, Maessen J, Dejong C, Kruitvvagen R, Slangen B. Diffusion of Enhanced Recovery principles in gynecologic oncology surgery: Is active implementation still necessary? Gynecol Oncol 2014; 134: 570-5.

57. Deniau B, Bouhadjari N, Faitot V, Mortazavi A, Kayem G, Mandelbrot L et al. Evaluation of a continuous improvement programme of enhanced recovery after caesarean delivery under neuraxial anaesthesia. ACCPM 2016; 35: 395-9.

58. Egerod I, Rud K, Specht K, Jensen PS, Trangbaæk A, Rønfelt I et al. Room for improvement in the treatment of hip fractures in Denmark. Dan Med Bull 2010; 57: A4199.

59. Francisco S, Batista S, Pena G. Fasting in Elective Surgical Patients: Comparison Amoung the Time Prescribed, Performed and Recommended on Peroperative Care Protocols. Arq Bras Cir Dig 2015; 28 : 250-4.

60. Hardt J, Schwarzbach M, Hasenberg T, Post S, Kienle P, Ronellenfitsch U. The effect of a clinical pathway for enhanced recovery of rectal resections on perioperative quality of care. Int $J$ Colorectal Dis 2013; 28: 1019-26. 
61. Hilal MA, Di Fabio F, Badran A, Alsaati H, Clarke H, Fecher I et al. Implementation of enhanced recovery programme after pancreatoduodenectomy: A single-centre UK pilot study. Pancreatology 2013; 13: 58-62.

62. Hubner M, Addor V, Slieker J, Griesser A, Lecureux E, Blanc C et al. The impact of an enhanced recovery pathway on nursing workload: A retrospective cohort study. Int J Surg 2015; 24: 45-50.

63. Hui V, Hyman N, Viscomi C, Osler T. Implementing a fast-track protocol for patients undergoing bowel resection: Not so fast. Am J Surg 2013; 206: 152-8.

64. Imbelloni L, Gomes D, Braga R, de Morais Filho G, da Silva A. Clinical strategies to accelerate recovery after surgery orthopedic femur in elderly patients. Anesth Essays Res 2014; 8: 156-61.

65. Kalogera E, Bakkum-Gamez J, Jankowski C, Trabuco E, Lovely J, Dhanorker S et al. Enhanced Recovery in Gynecologic Surgery. Obstet Gynecol 2013; 122: 319-28.

66. Kolozsvari N, Capretti G, Kaneva P, Neville A, Carli F, Liberman S et al. Impact of an enhanced recovery program on short-term outcomes after scheduled laparoscopic colon resection. Surg Endosc 2013; 27: 133-8.

67. Labgaa I, Jarrar G, Joliat G-R, Allemann P, Gander S, Blanc C et al. Implementation of Enhanced Recovery (ERAS) in Colorectal Surgery Has a Positive Impact on Non-ERAS Liver Surgery Patients. World J Surg 2016; 40: 1082-91.

68. Maessen J, Hoff C, Jottard K, Kessels A, Bremers A, Havenga K et al. To eat or not to eat: Facilitating early oral intake after elective colonic surgery in the Netherlands. Clin Nutr 2009; 28: 2933.

69. Miller T, Thacker J, White W, Mantyh C, Migaly J, Jin J et al. Reduced Length of Hospital Stay in Colorectal Surgery after Implementation of an Enhanced Recovery Protocol. Anesth Analg 2014; 118: 1052-61.

70. Schwarzbach $\mathrm{M}$, Hasenberg $\mathrm{T}$, Linke $\mathrm{M}$, Kienle $\mathrm{P}$, Post $\mathrm{S}$, Ronellenfitsch U. Perioperative quality of care is modulated by process management with clinical pathways for fast-track surgery of the colon. Int J Colorectal Dis 2011; 26: 1567-75.

71. Selby L, Rifkin M, Yoon S, Ariyan C, Strong V. Decreased length of stay and earlier oral feeding associated with standardized postoperative clinical care for total gastrectomies at a cancer center. Surgery 2016; 160: 607-12.

72. Thompson E, Gower S, Beilby D, Wallace S, Tomlinson S, Guest G et al. Enhanced Recovery after Surgery program for elective abdominal surgery at three Victorian hospitals. Anaesth Intens Care 2012; 40: 450-9.

73. Zargar-Shoshtari K, Connolly A, Israel L, Hill A. Fast-track surgery may reduce complications following major colonic surgery. Dis Colon Rectum 2008; 51: 1633-40.

74. Hancock S, Cresci G, Martindale R. The clear liquid diet: when is it appropriate? Curr Gastroenterol Rep 2002; 4: 324-31.

75. Warren J, Bhalla V, Cresci G. Postoperative diet advancement: Surgical dogma vs evidencebased medicine. Nutr Clin Pract 2011; 26: 115-25.

76. Sriram K, Ramasubramanian V, Meguid MM. Special postoperative diet orders: Irrational, obsolete, and imprudent. Nutrition 2016; 32: 498-502.

77. Lau C, Phillips E, Bresee C, Fleshner P. Early use of low residue diet is superior to clear liquid diet after elective colorectal surgery: a randomized controlled trial. Ann surg 2014; 260: 641-94.

78. Jeffery KM, Harkins B, Cresci G, Martindale R. A clear liquid diet is no longer a necessity in the routine postoperative management of surgical patients. Int J Orthop Trauma Nurs 1996; 2: 94-5.

79. Rattray M, Desbrow B, Roberts S. Comparing nutritional requirements, provision and intakes among patients prescribed therapeutic diets in hospital: an observational study. Nutrition 2017; In Press.

80. Masood SN, Siddiqui IA, Masood MF, Masood Y, Mumtaz SN. To assess the practices about initiation of oral maternal feeding after cesarean section under regional anesthesia. Pak J Med Sci 2011; 27: 996-1000. 
81. Carey SK, Conchin S, Bloomfield-Stone S. A qualitative study into the impact of fasting within a large tertiary hospital in Australia - the patients' perspective. J Clin Nurs 2015; 24: 1946-54.

82. Van Wijk MGF, Smalhout B. A postoperative analysis of the patient's view of anaesthesia in a Netherlands' teaching hospital. Anaesthesia 1990; 45: 679-82.

83. Gan TJ, Sloan F, De L Dear G, El-Moalem HE, Lubarsky DA. How much are patients willing to pay to avoid postoperative nausea and vomiting? Anesth Analg 2001; 92: 393-400.

84. Yeung SE, Fenton TR. Colorectal surgery patients prefer simple solid foods to clear fluids as the first postoperative meal. Dis Colon Rectum 2009; 52: 1616-23.

85. Gan TJ, Diemunsch P, Habib AS, Kovac A, Kranke P, Meyer TA et al. Consensus Guidelines for the Management of Postoperative Nausea and Vomiting. Anesth Analg 2014; 118: 85-113.

86. Morley J. Decreased food intake with aging. Gerontol A Biol Sci Med Sci 2001; 56: 81-8.

87. Trastulli S, Cirocchi R, Listorti C, Cavaliere D, Avenia N, Gullà N et al. Laparoscopic vs open resection for rectal cancer: a meta-analysis of randomized clinical trials. Colorectal Dis 2012; 14: e277-e96. 